\title{
Robust Modal Damping Control for Active Flutter Suppression
}

\author{
Julian Theis * \\ Hamburg University of Technology, Hamburg, Germany, 21073 \\ Harald Pfifer ${ }^{\dagger}$ \\ University of Nottingham, Nottingham, United Kingdom, NG7 2RD \\ Peter Seiler \\ University of Minnesota, Minneapolis, MN 55455
}

\begin{abstract}
Flutter is an unstable oscillation caused by the interaction of aerodynamics and structural dynamics. It is current practice to operate aircraft well below their open-loop flutter speed in a stable flight regime. For future aircraft, weight reduction and aerodynamically efficient high aspect ratio wing design reduce structural stiffness and thus reduce flutter speed. Active control of the flutter phenomena can counter adverse aeroservoelastic effects and allow operation of an aircraft beyond its open-loop flutter speed. This paper presents a systematic robust control design method for active flutter suppression. It extends the standard four block mixed sensitivity formulation by a means to target specific dynamic modes and add damping. This enables a control design to augment damping of critical flutter modes with minimal impact on the rigid-body autopilots. Finally, the design scheme uses a manageably low number of tunable parameters with a clear physical interpretation. Tuning the controller is hence considerably easier than with standard approaches. The method is demonstrated by designing an active flutter suppression controller for a small, flexible unmanned aircraft and verified in simulation.
\end{abstract}

\section{Nomenclature}

$\begin{array}{ll}a & =\text { acceleration } \\ A, B, C, D & =\text { state space matrices } \\ d & =\text { disturbance } \\ i & =\text { index variable } \\ \mathrm{j} & =\text { imaginary unit } \\ F & =\text { vector of forces } \\ \mathcal{F} & =\text { vector of generalized forces }\end{array}$

\footnotetext{
*Postdoctoral Research Associate, Institute of Aircraft Systems Engineering, Nesspriel 5, 21129 Hamburg, Germany.

$\dagger$ Assistant Professor, Department of Mechanical, Materials and Manufacturing Engineering, University Park Campus, Nottingham, NG7 2RD, United Kingdom.

${ }^{\ddagger}$ Associate Professor, Aerospace Engineering and Mechanics Department, Akerman Hall, 110 Union St SE, Minneapolis, MN 55455, USA.
} 


$$
\begin{aligned}
& g \quad=\text { gravitational constant } \\
& G(s), H(s)=\text { transfer functions } \\
& \text { I } \quad \text { identity matrix } \\
& J \quad=\text { inertia tensor } \\
& \text { K }=\text { controller } \\
& L_{1}, \ldots, L_{4}=\text { left flap deflections } \\
& m=\text { mass } \\
& M \quad=\quad \text { vector of moments } \\
& \mathcal{M}, \mathcal{D}, \mathcal{K}=\text { generalized mass, damping, and stiffness matrices } \\
& \hat{M}(s) \quad=\text { modal velocity transfer function } \\
& M(s), N(s)=\text { modal transfer function } \\
& n=\text { noise, } \text { count } \\
& p \quad=\text { performance output vector of dynamic system, roll rate } \\
& P \quad=\text { plant model } \\
& q \quad=\text { pitch rate } \\
& r \quad=\quad \text { reference signal, position vector } \\
& R_{1}, \ldots, R_{4} \quad=\text { right flap deflections } \\
& s \quad=\quad \text { Laplace variable } \\
& u \quad=\text { control signal } \\
& v, w \quad=\quad \text { input vector of dynamic system } \\
& V \quad=\text { static weight } \\
& V_{\infty} \quad=\text { trim airspeed } \\
& W \quad=\quad \text { dynamic weight } \\
& w \quad=\quad \text { vertical velocity } \\
& x \quad=\quad \text { state vector of dynamic system } \\
& y, z=\text { output vector of dynamic system } \\
& \alpha=\text { tuning parameter for input disturbance } \\
& \delta=\text { control surface deflections } \\
& \zeta \quad=\text { damping ratio } \\
& \eta \quad=\quad \text { generalized displacement of structural mode } \\
& \lambda \quad=\text { eigenvalue } \\
& \xi \quad=\text { modal state vector }
\end{aligned}
$$




$$
\begin{array}{ll}
\bar{\sigma} & =\text { largest singular value } \\
\varphi & =\text { left eigenvector } \\
\omega & =\text { rotational rates, frequency } \\
\omega_{0} & =\text { natural frequency } \\
\mathbb{C} & =\text { field of complex numbers } \\
\mathbb{R} & =\text { field of real numbers } \\
\operatorname{Re} & =\text { real part } \\
\operatorname{Im} & =\text { real part }
\end{array}
$$

Subscripts

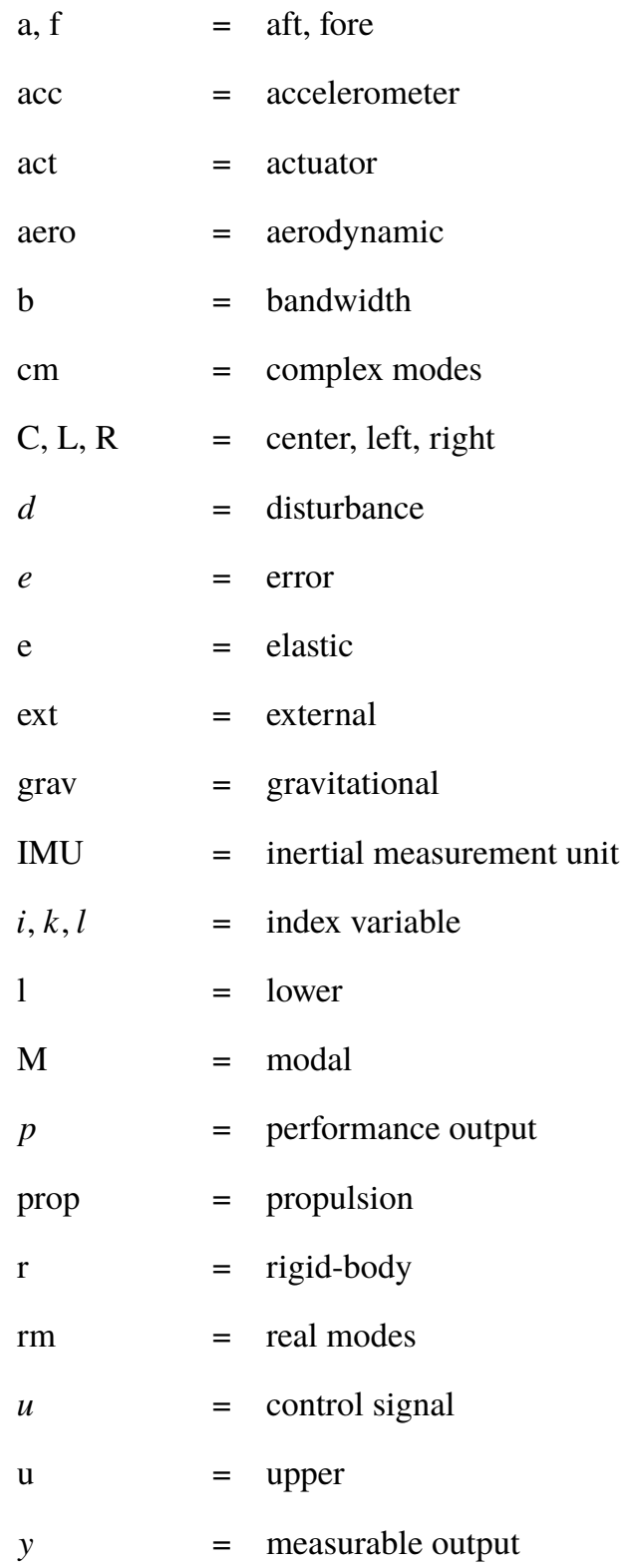




\section{Introduction}

Aeroelastic flutter involves the adverse interaction of aerodynamics with structural dynamics and potentially rigid-body dynamics. It produces an unstable oscillation that often results in structural failure as shown in the picture sequence in Figs. 1 $1 \mathrm{a}-\mathrm{e}$. Conventional aircraft are designed in accordance with FAA/EASA certification specification 25.629 such that flutter does not occur within the range of their operating conditions. This is usually achieved through the use of stiffening structure and thus at the expense of additional mass. The use of active control systems to expand the flutter boundary could therefore lead to a decrease in structural mass and consequently increase fuel efficiency and performance for future aircraft [1]. The fundamental principles of active flutter suppression control are discussed in [2, 3] and a comprehensive survey was recently provided in [4].

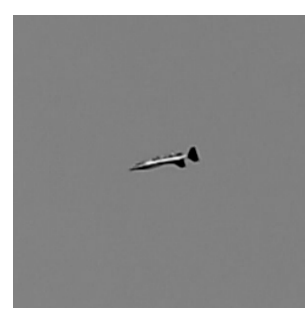

(a)

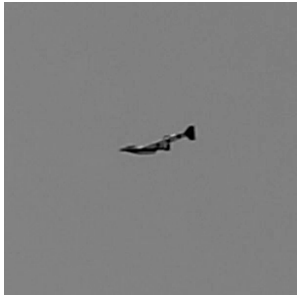

(b)

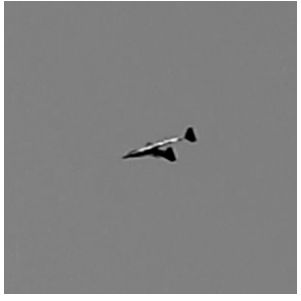

(c)

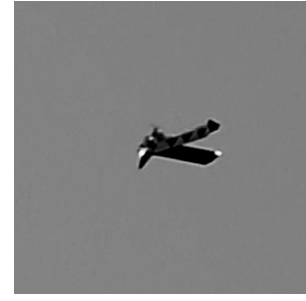

(d)

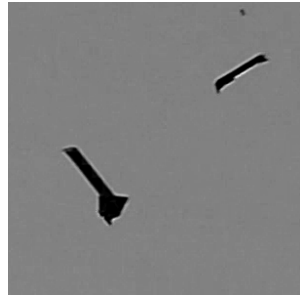

(e)

Fig. 1 Open-loop flutter and catastrophic failure during a flight test at the University of Minnesota on August 25th 2015. The sequence shows pictures taken in a one second interval.

Early research on active flutter suppression relied to a large extent on what is known as collocated feedback. Collocated feedback employs sensors and actuators in the same location, see e. g. [5]. Collocated controllers are, in general, easy to design using root-locus techniques and are extremely robust with respect to gain variation. A closely related approach, termed the concept of identically located force and acceleration in [6], was successfully applied to address the damping of structural modes on the B-1 aircraft [7, 8]. A similar configuration was also used in the first flight test beyond flutter speed, conducted in 1973 on a modified B-52 aircraft [9]. The control system on that aircraft involved two single feedback loops that fed back filtered vertical acceleration signals, acquired on the wing, to control surfaces located nearby (outboard ailerons and flaperons). Collocated acceleration feedback is also proposed in various other publications concerned with flutter suppression, e. g. [10--15].

The other main branch of research on flutter suppression control considers multivariable optimal control techniques such as linear quadratic Gaussian (LQG) 16-18] as well as $\mathcal{H}_{\infty}$-norm optimal and linear parameter-varying (LPV) control [19-24]. Recent flight test demonstrations of active flutter suppression, e. g., on Lockheed Martin's Body Freedom Flutter vehicle [23], make use of such techniques. Multivariable controllers can make optimal use of all available control surfaces and sensor signals without potentially restrictive pre-grouping of inputs and outputs. As a drawback, accurate models are required and formulating an adequate optimization objective that represents the actual control design objectives can become complicated. Another multivariable approach that has recently emerged can be 
classified as modal isolation. It seeks to target specific dynamic modes, such as the aeroelastic flutter mode, and avoid interaction with other dynamics. This is achieved by first calculating a signal that represents a modal state. Then, a feedback loop is closed with this signal [25-27].

This paper contributes a systematic $\mathcal{H}_{\infty}$-control design framework for modal damping and mode attenuation. Inspired by [28] and [25], specific dynamic modes are incorporated as a performance measure. Section II] formulates the method as a generalization of the standard four-block problem for control design. The objective of adding damping to a specific mode is expressed as reducing peaks in the frequency response of a closed-loop transfer function. The $\mathcal{H}_{\infty}$ closed loop shaping method augments the controller with roll-off and wash-out filters such that control activity can be precisely confined to a specific frequency range. Doing so avoids undesired interaction with unmodeled or uncertain plant dynamics at high frequencies and with autopilot systems at low frequencies. The proposed design method is hence particularly suitable for flutter suppression control. This is demonstrated by applying the method to the flight-test-validated model of a small, remote-piloted aircraft, described in Section [II] The model involves asymmetric wing stiffness, causing non-negligible coupling of longitudinal and lateral dynamics. Further, two different flutter modes are present, which challenges conventional control methods. A comprehensive control design study that addresses simultaneous stabilization of these two flutter modes and verification in simulation is provided in Sec. IV This paper builds on prior work in [29, 30] with three main extensions. Firstly, a novel parameterization for all involved weighting functions is provided. This parameterization has a manageably low number of tunable parameters with clear physical interpretations and is hence easy to apply. In addition, the paper provides specific guidelines on how these parameters are chosen. Secondly, an explicit formulation for including generalized velocities of dynamic modes as a performance measure is provided. Finally, significant engineering insight about the application of the method is added through the design example and verification in a simulation that includes actuator nonlinearities such as rate limits and backlash.

\section{Control Design Methodology}

Shaping closed-loop transfer functions through $\mathcal{H}_{\infty}$-norm optimization is a well-established approach for control design [31, 32]. The $\mathcal{H}_{\infty}$-norm of a dynamic system $H(s)$ from input $w$ to output $z$ is defined as

$$
\|H(s)\|=\sup _{\omega} \bar{\sigma}(H(\mathrm{j} \omega))=\sup _{w \in \mathcal{L}_{2} \backslash\{0\}} \frac{\|z\|_{2}}{\|w\|_{2}},
$$

where $\bar{\sigma}(\cdot)$ denotes the largest singular value. This norm measures the maximum gain of the transfer function $H(s)$, i. e., the largest amplification of $\mathcal{L}_{2}$ input signals over all frequencies and input/output directions. In closed loop shaping, the $\mathcal{H}_{\infty}$-norm is used to characterize performance in terms of a bound on the magnitude of closed-loop transfer functions. These bounds are specified by the control designer through frequency-dependent weights, leading to a so-called generalized plant. Well established algorithms exist that synthesize a dynamic controller for a given 
generalized plant. The resultant stabilizing controller minimizes an upper bound on the $\mathcal{H}_{\infty}$-norm of the weighted closed-loop transfer functions. This upper bound multiplied by the inverse of the weights bounds the magnitude of the unweighted closed-loop transfer functions and hence guarantees their shape. Standard $\mathcal{H}_{\infty}$-controller synthesis algorithms solve Ricatti equations or use convex optimization [33-35]. These algorithms provide an optimal controller that is, however, of the same order as the generalized plant. If additional structure is imposed on the controller, e. g. a PID controller or fixed-order filter is required, non-smooth and non-convex optimization methods can be used instead [36-38]. Doing so only changes the controller synthesis step, not the design formulation. As such, the method presented in this paper is also applicable for structured $\mathcal{H}_{\infty}$-controller design. With mature synthesis machinery for both full-order and structured controllers readily available, e. g., in the Matlab Robust Control Toolbox[39], the challenging part of any $\mathcal{H}_{\infty}$-design is to provide meaningful performance specifications in terms of a generalized plant.

\section{A. Generalized Plant for Closed-Loop Shaping}

In the following, the closed-loop transfer functions that ought to be shaped by the $\mathcal{H}_{\infty}$-controller and the weights that specify their desired shapes are introduced. Consider a dynamic system

$$
P=\left[\begin{array}{l}
P_{p} \\
P_{y}
\end{array}\right]:\left\{\begin{array}{l}
\dot{x}=A x+B v \\
p=C_{p} x+D_{p} v \\
y=C x+D v
\end{array}\right.
$$

with input $v(t) \in \mathbb{R}^{n_{v}}$, state vector $x(t) \in \mathbb{R}^{n_{x}}$, a measurable output $y(t) \in \mathbb{R}^{n_{y}}$, and an additional performance output $p(t) \in \mathbb{R}^{n_{p}}$. The state space matrices $(A, B, C, D)$ represent a given plant model while $\left(C_{p}, D_{p}\right)$ can be freely chosen to define an output of interest $p$. Through this choice, any state variable or linear combination of state variables can be included in the problem formulation analogous to standard LQ-optimal control. Let $P$ be interconnected with a controller $K$ as shown in Fig. 2. All measurable outputs $y$ are subject to noise $n$ and constitute the feedback loop. A reference $r(t) \in \mathbb{R}^{n_{y}}$ enters the loop. This reference is assumed to have the form $r=\left[\begin{array}{c}r_{1}(t) \\ 0\end{array}\right]$ with $r_{1}(t) \in \mathbb{R}^{n_{r}}$. That is, it is assumed without loss of generality that the first $n_{r} \leq n_{y}$ outputs are tracked and that the remaining $n_{y}-n_{r}$ outputs are additional feedback signals. Note that the output $p$ is not part of the feedback loop and does not need to be measurable.

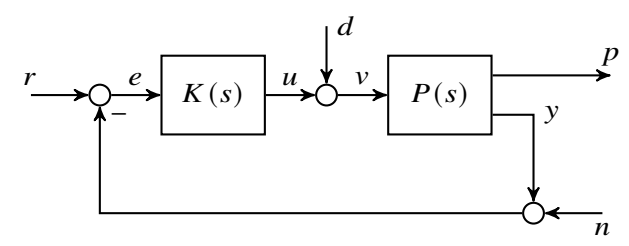

Fig. 2 Standard closed loop with additional performance output. 
Define the output sensitivity $S_{\mathrm{o}}=\left(I+P_{y} K\right)^{-1}$ and input sensitivity $S_{\mathrm{i}}=\left(I+K P_{y}\right)^{-1}$. The transfer functions governing the closed-loop interconnection are

$$
\left[\begin{array}{l}
e \\
u \\
p
\end{array}\right]=\left[\begin{array}{cc}
S_{\mathrm{o}} & -P_{y} S_{\mathrm{i}} \\
K S_{\mathrm{o}} & -K P_{y} S_{\mathrm{i}} \\
P_{p} K S_{\mathrm{o}} & P_{p} S_{\mathrm{i}}
\end{array}\right]\left[\begin{array}{c}
r-n \\
d
\end{array}\right]
$$

For $p=y$ (and hence $P_{p}=P_{y}$ ), Eq. (3) collects the six fundamental closed-loop transfer functions of a multivariable feedback loop [32]. Shaping the transfer function from $\left[\begin{array}{c}r-n \\ d\end{array}\right]$ to $\left[\begin{array}{c}e \\ u\end{array}\right]$ is commonly referred to as the four block problem [40]. The four block problem yields controllers with desireable robustness properties and captures all important performance aspects of control design, i. e., tracking $\left(S_{\mathrm{o}}\right)$, disturbance attenuation $\left(P_{y} S_{\mathrm{i}}\right)$, and control effort limitation $\left(K S_{\mathrm{o}}\right.$ and $\left.K P_{y} S_{\mathrm{i}}\right)$. Therefore, it is used as the basis for the method in this paper. The output $p=y$ is not used in the standard four block problem, since the complimentary output sensitivity $P_{y} K S_{\mathrm{o}}=I-S_{\mathrm{o}}$ is already implicitly considered through $S_{\mathrm{o}}$. In addition, $P_{y} S_{\mathrm{i}}$ already appears as the map from $d$ to $e$. While adding $y$ offers no distinct advantages, considering a fictitious performance output $p$ is a straightforward yet highly beneficial generalization of the four block problem. An appropriate choice of $p$ can emphasize specific dynamics in the output, e.g., velocity states [41] and modal velocities as shown in Sec. III.C The output $p$ thus provides additional means to specify control objectives such as damping augmentation. Note that a generalized formulation for two-degrees-of-freedom controllers and arbitrary disturbance models can be found in [42].

The generalized closed loop shown in Fig. 3 is used to formulate loop shape requirements. Its transfer function is

$$
\left[\begin{array}{l}
z_{1} \\
z_{2} \\
z_{3}
\end{array}\right]=\left[\begin{array}{ccc}
W_{e}(s) V_{e}^{-1} & 0 & 0 \\
0 & W_{u}(s) V_{u}^{-1} & 0 \\
0 & 0 & W_{p}(s) V_{p}^{-1}
\end{array}\right]\left[\begin{array}{cc}
S_{\mathrm{o}} & -P_{y} S_{\mathrm{i}} \\
K S_{\mathrm{o}} & -K P_{y} S_{\mathrm{i}} \\
P_{p} K S_{\mathrm{o}} & P_{p} S_{\mathrm{i}}
\end{array}\right]\left[\begin{array}{cc}
V_{e} & 0 \\
0 & V_{d}
\end{array}\right]\left[\begin{array}{c}
w_{1} \\
w_{2}
\end{array}\right] .
$$

The weights $W_{e}(s), W_{u}(s), W_{p}(s), V_{e}, V_{u}, V_{d}$, and $V_{p}$ express performance objectives for the controller synthesis by imposing desired loop shapes as detailed in Sec. II.B Each of the weights has non-zero entries only on the diagonal, such that physically interpretable inputs and outputs are weighted, which considerably simplifies design choices. The weighting filters $W_{e}(s), W_{u}(s), W_{p}(s)$ are frequency-dependent, while $V_{e}, V_{u}, V_{p}, V_{d}$ denote static weights. This separation is non-standard in $\mathcal{H}_{\infty}$-control. It reduces the number of tuning parameters compared to an arbitrary signal-weighted design, while retaining engineering insight. Text books, e. g. [32], implicitly make use of static weights by scaling the model prior to designing a controller. Doing so can obscure the influence such scalings have on the performance specifications, which can complicate tuning. The structure of the weights in Eq. (4) makes this influence 
explicit. As shown in the subsequent Section III.B initial guesses for the weights $V$ are easily found as maximum acceptable values for errors and control signals, similar to the normalizing scalings of Skogestad \& Postlethwaite [32] and Bryson's rule from LQG control [43]. The frequency-dependent weighting filters can be chosen independent of the plant model's units to represent closed-loop bandwidth and magnitude bounds.

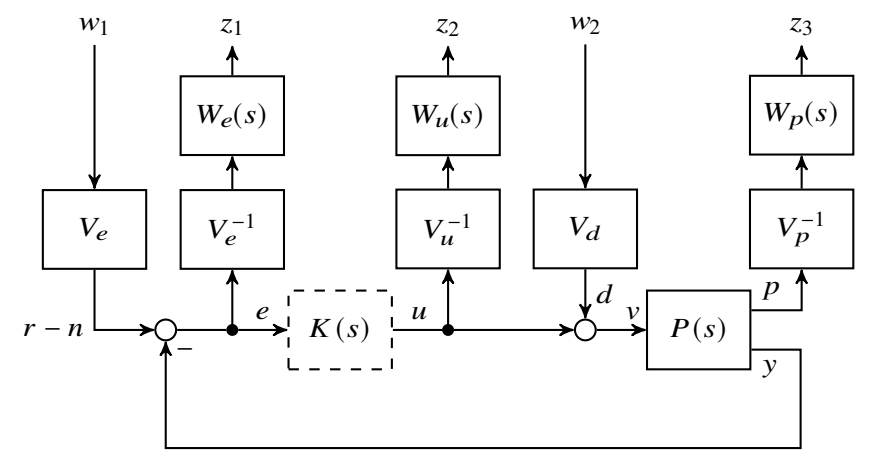

Fig. 3 Generalized closed loop for control design.

\section{B. Weight Selection}

This subsection discusses a parameterization of the weights and how it is used for tuning. The discussion considers a representative example with two control inputs and two measured outputs. The first output shall be tracked, whereas the second output is used as an additional feedback signal. Further, only the first control input may be used in steady state. The second shall be restricted to a specific frequency range. This example covers sufficient complexity to be adaptable to a large variety of control design problems including flutter control design.

The weighting filter $W_{e}$ multiplies the outputs of $V_{e}^{-1} S_{\mathrm{o}} V_{e}$ and $-V_{e}^{-1} P_{y} S_{\mathrm{i}} V_{d}$ in Eq. (4). Thus the choice of this weighting filter determines the shape of the sensitivity $S_{\mathrm{o}}$ and disturbance sensitivity $P_{y} S_{\mathrm{i}}$. It represents design specifications on sensitivity reduction and is parameterized as

$$
W_{e}(s)=\left[\begin{array}{cc}
\frac{s+\omega_{\mathrm{b}} \sqrt{\frac{3}{1-\epsilon^{2}}}}{2 s+\epsilon \omega_{\mathrm{b}} \sqrt{\frac{3}{1-\epsilon^{2}}}} & 0 \\
0 & 0.5
\end{array}\right]
$$

The first entry $W_{e, 1}(s)$ in Eq. (5) has the properties $W_{e, 1}(0)=\frac{1}{\epsilon},\left|W_{e, 1}\left(\mathrm{j} \omega_{\mathrm{b}}\right)\right|=1$, and $W_{e, 1}(\infty)=0.5$, where $\epsilon \ll 1$ denotes the desired steady-state error constant. Its frequency response is shown in Fig. 4 For the tracked output, it has integral behavior up to the desired closed-loop bandwidth $\omega_{\mathrm{b}}$ and gain 0.5 beyond that frequency. The second output is a constant gain $W_{e, 2}(s)=0.5$. By choosing the static weights $V_{e}$ and $V_{d}$, bounds on the closed-loop transfer functions as shown in Figs. 5 and 6 are implied. That is, the weights dictate a sensitivity reduction up to $\omega_{\mathrm{b}}$ and limit sensitivity degradation beyond that frequency to a factor of two in the tracked output, see Fig. 5 For the second output, sensitivity 
should not exceed two. Note that a peak sensitivity of less than two implies a gain margin of at least $6 \mathrm{~dB}$, see e. g. [32]. The choice of $V_{e}$ determines cross-coupling and leaves the specifications on the diagonal unaffected. Consequently, $V_{e}, 1$ can be selected as the maximum expected command value $r_{1}$ for the tracked output and $V_{e, 2}$ as the maximum acceptable cross-coupling due to this command. Further, Fig. 6 shows that the relative size of $V_{e}$ and $V_{d}$ specifies the level of disturbance attenuation.

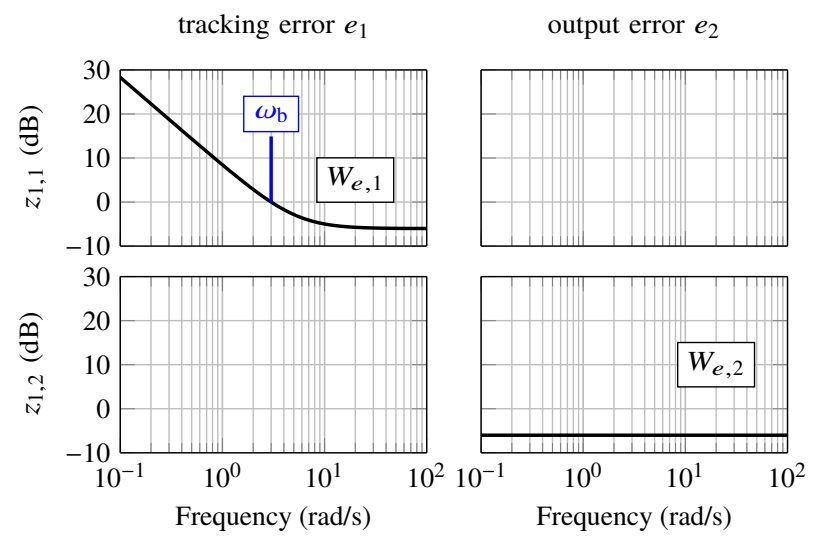

Fig. 4 Parameterization for weight $W_{e}$.

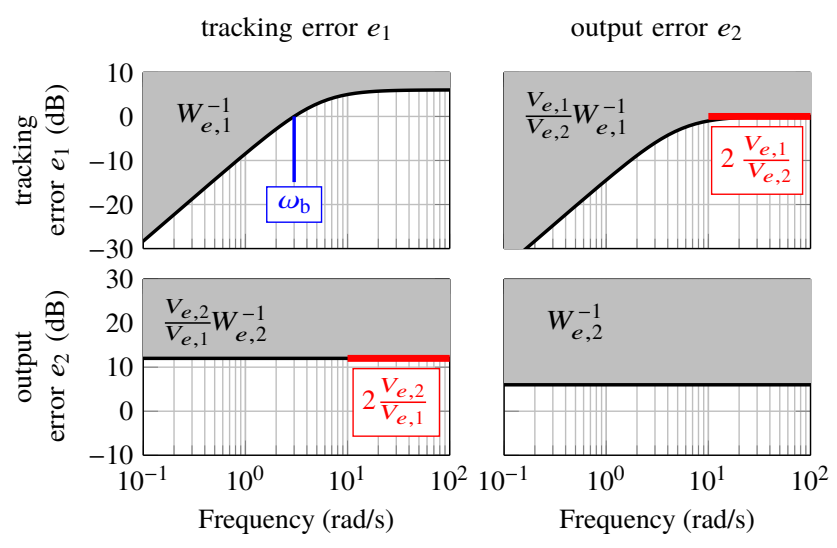

Fig. 5 Implied loop shape requirements on the sensitivity $S_{0}$.

The weighting filter $W_{u}(s)$ determines the shape of the control sensitivity $K S_{\mathrm{o}}$ and hence distributes control authority across frequency. It is parameterized as

$$
W_{u}(s)=\left[\begin{array}{ccc}
\frac{s+\omega_{\mathrm{u}, 1}}{0.01 s+\omega_{\mathrm{u}, 1}} & 0 \\
0 & \frac{s+\omega_{\mathrm{l}, 2}}{s+0.01 \omega_{\mathrm{l}, 2}} & \frac{s+\omega_{\mathrm{u}, 2}}{0.01 s+\omega_{\mathrm{u}, 2}}
\end{array}\right] .
$$

Here, $\omega_{\mathrm{u}, 1}$ sets the upper available bandwidth for the control signals $u_{1}$, while $\omega_{\mathrm{u}, 2}$ and $\omega_{1,2}$ specify upper and lower limits for $u_{2}$. The corresponding frequency response is shown in Fig. 7. The weight on $u_{1}$ has unit gain up to $\omega_{\mathrm{u}, 1}$ and 
disturbance $d_{1}$
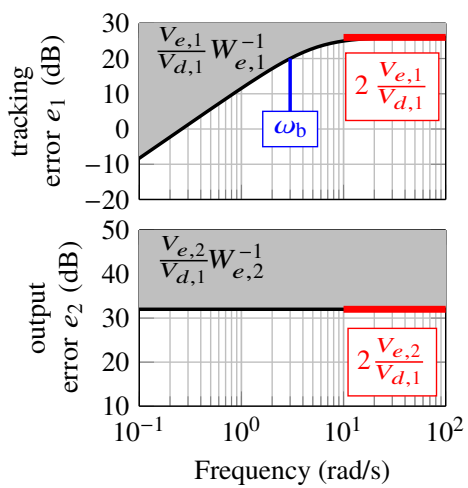

disturbance $d_{2}$
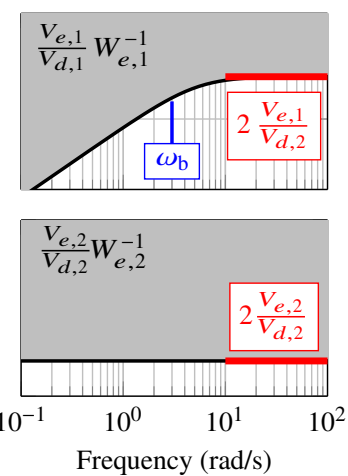

Fig. 6 Implied loop shape requirements on the load disturbance sensitivity $P_{y} S_{\mathrm{i}}$.

differentiating behavior beyond that frequency. The weight on $u_{2}$ has approximately unit gain between $\omega_{1,2}$ and $\omega_{\mathrm{u}, 2}$, and increasing gain outside that frequency range. This choice, in conjunction with a choice for $V_{u}$ and $V_{d}$, implies the

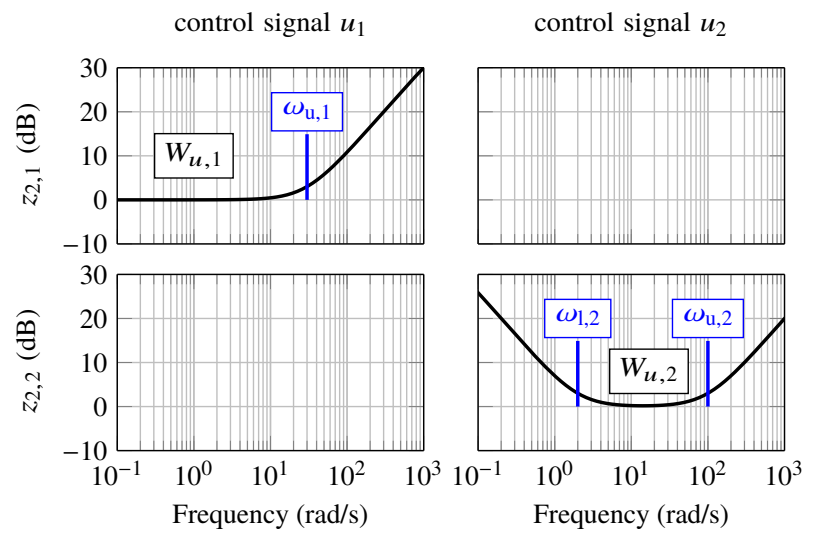

Fig. 7 Parameterization for weight $W_{u}$.

bounds shown in Figs. 8 and 9 At high frequencies, a low magnitude in $K S_{\mathrm{o}}$ is only achieved when $K$ rolls off, since $S_{\mathrm{o}} \approx I$ as $P_{y} K \rightarrow 0$. Therefore, the roll-off requirement on $K S_{\mathrm{o}}$ also imposes a roll-off on the controller $K$ and reduces the control activity beyond $\omega_{\mathrm{u}, 1}$ and $\omega_{\mathrm{u}, 2}$ for $u_{1}$ and $u_{2}$, respectively. Such a roll-off is necessary to avoid excitation of unmodeled or uncertain dynamics at high frequencies and to reduce control action in response to noise. Similarly, the requirement that $K P_{y} S_{\mathrm{i}}=I-S_{\mathrm{i}}$ has low magnitude in the low frequency range implies that $S_{\mathrm{i}} \approx I$ and hence is only achieved with a low magnitude in $K$. The wash-out requirement on $K P_{y} S_{\mathrm{i}}$ hence translates directly to $K$ and confines the control activity for $u_{2}$ to frequencies above $\omega_{1,2}$. A bandpass characteristic limits interaction with low-frequency dynamics and is particularly useful for flutter control cf. [21].

Figure 8 shows that the ratio of $V_{e}$ to $V_{u}$ bounds the maximum controller gain. The weight $V_{u}$ is consequently selected to represent available control action relative to the previously specified maximum errors. Thus, $V_{u}$ is often selected based on the actual saturation limits of the actuators. Figure 9 finally shows the transfer functions that represent 
the control effort in response to disturbances. The ratio of $V_{u}$ and $V_{d}$ determines the maximum gain. Thus, $V_{d}$ can be selected based on known maximum disturbances. It is clear that $V_{d, i} \leq V_{u, i}$ for a sensible design, as the maximum input disturbance must be smaller or equal to the available control signal. A simple choice is $V_{d}=\alpha V_{u}$ with $0<\alpha \leq 1$, which assumes that disturbances at all inputs are a fixed percentage of the maximum inputs. Thus, $\alpha$ has the interpretation of input uncertainty and can be used as an alternative tuning parameter instead of $V_{d}$.

tracking error $e_{1}$
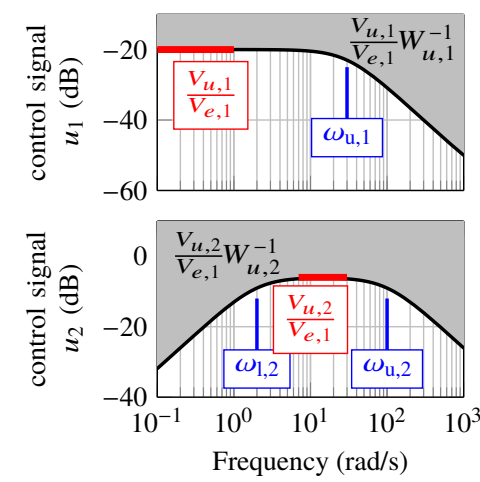

output error $e_{2}$
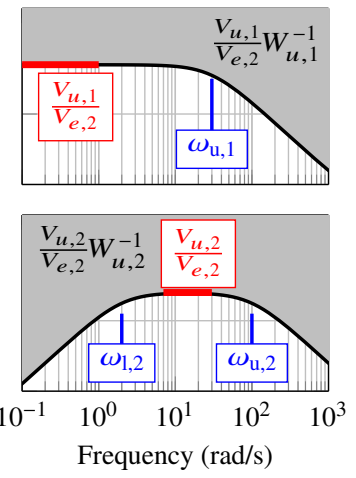

Fig. 8 Implied loop shape requirements on the control sensitivity $K S_{0}$.

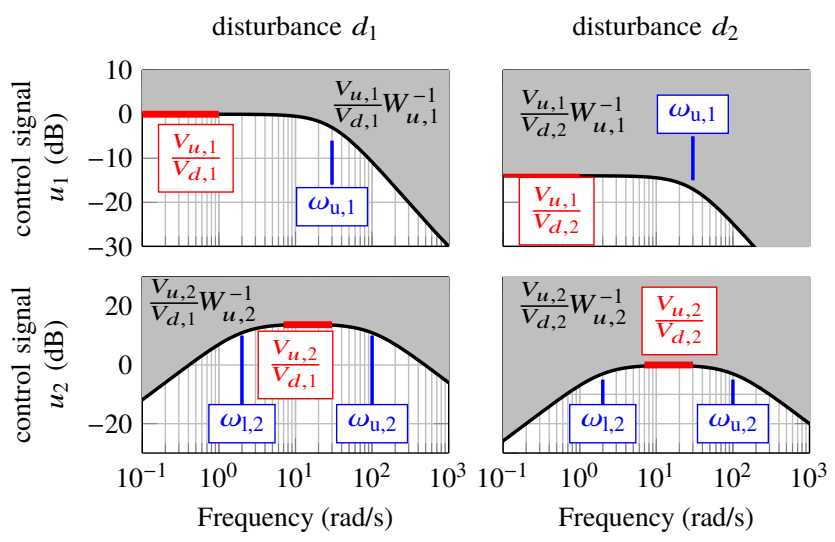

Fig. 9 Implied loop shape requirements on the input complementary sensitivity $K P_{y} S_{\mathrm{i}}$.

In summary, the proposed architecture has a manageably low total number of tunable parameters ( 9 or 10 for the present example, depending on whether $V_{d}=\alpha V_{u}$ is used). One critique of $H_{\infty}$-control, especially for signal-weighted design schemes, is that there are many parameters with opaque interdependence that must be chosen to get a reasonable controller [32]. In the proposed formulation, each of the parameters has a clear interpretation. The weighting filters $W_{e}(s)$ and $W_{u}(s)$ represent bandwidth requirements and usually require little adjustment. Tuning is mainly performed through the static weights $V_{e}, V_{u}$, and $V_{d}$ (or $\alpha$ ) and thus with only $n_{y}+2 n_{u}$ (or $\left.n_{y}+n_{u}+1\right)$ parameters. The interpretation as maximum values for control errors, control signals, and disturbances yields good initial tuners for these weights. This approach resembles Bryson's rule, commonly used to obtain initial tuners for LQG design weights as the inverse of 
maximum acceptable values [43]. For comparison, standard LQG control with diagonal weighting matrices requires a state feedback and a Kalman filter design. The state feedback design uses $n_{x}+n_{u}$ or $n_{y}+n_{u}$ parameters, depending on whether states or outputs are penalized. Similarly, the Kalman filter design requires $n_{y}+n_{x}$ or $n_{y}+n_{u}$ parameters, depending on whether process noise or input disturbances are modeled.

It remains to discuss the selection of $W_{p}$ and $V_{p}$. How these weights are chosen depends to a larger extend on the specific choice of the output variables $p$, but often $W_{p}=I$ is sufficient and $V_{p}$ is selected such that $p$ is normalized to a magnitude of 1 . The subsequent section II.C details the case where $p$ is used to target a specific dynamic mode of the system and augment its damping.

\section{Adding Modal Damping via Modes Targeting}

It is shown in the following that damping can be effectively augmented by penalizing the generalized velocity of a dynamic mode. Dynamic modes are decoupled subsystems of order one (real) or two (complex) such that the system $P_{y}(s)$ can be expressed as

$$
P_{y}(s)=D+\sum_{k=1}^{n_{\mathrm{cm}}} M_{k}(s)+\sum_{l=1}^{n_{\mathrm{rm}}} N_{l}(s) .
$$

The $n_{\mathrm{rm}}$ real modes $N_{l}(s)$ are not considered further in this section as they do not contribute to oscillations. The $n_{\mathrm{cm}}$ complex modes $M_{k}(s)$ contain oscillatory system dynamics. They can be represented by state space realizations

$$
M_{k}:\left\{\begin{array}{l}
{\left[\begin{array}{l}
\dot{\xi}_{k} \\
\ddot{\xi}_{k}
\end{array}\right]=\left[\begin{array}{cc}
0 & 1 \\
-\omega_{0, k}^{2} & -2 \zeta_{i} \omega_{0, k}
\end{array}\right]\left[\begin{array}{c}
\xi_{k} \\
\dot{\xi}_{k}
\end{array}\right]+\left[\begin{array}{c}
B_{1, k} \\
B_{2, k}
\end{array}\right] v \quad k=1, \ldots, n_{\mathrm{cm}} .} \\
y_{M, k}=\left[\begin{array}{ll}
C_{1, k} & C_{2, k}
\end{array}\right]\left[\begin{array}{c}
\xi_{k} \\
\dot{\xi}_{k}
\end{array}\right]
\end{array}\right.
$$

where $\omega_{0, k}$ is the natural frequency and $\zeta_{k}$ is the damping ratio of the $k^{\text {th }}$ dynamic mode. In this form, the modal state variables are a generalized displacement $\xi_{k}$ and a generalized velocity $\dot{\xi}_{k}$. The row vectors $B_{1, k} \in \mathbb{R}^{1 \times n_{v}}$ und $B_{2, k} \in \mathbb{R}^{1 \times n_{v}}$ describe how the input $v$ excites the $k^{\text {th }}$ mode.

Assume that $B_{1, k}=[0 \cdots 0]$ which is the case, e. g., for ordinary mechanical systems [44]. The multi-input-singleoutput transfer function $\hat{M}_{k}(s)$ from $v$ to $\dot{\xi}_{k}$ in this case is

$$
\hat{M}_{k}(s)=\frac{s}{s^{2}+2 \zeta_{k} \omega_{0, k} s+\omega_{0, k}^{2}} B_{2, k}
$$

It has a distinct peak at the frequency $\omega_{0, k}$ and exhibits band-pass characteristics, see Fig. 10. For underdamped modes 
with $\zeta<\frac{1}{\sqrt{2}}$, the damping ratio $\zeta$ is inversely proportional to the peak magnitude $\|\hat{M}\|$ as

$$
\zeta_{k}=\frac{1}{\left\|\hat{M}_{k}\right\|} \frac{\bar{\sigma}\left(B_{2, k}\right)}{2 \omega_{0, k}}
$$

Hence, reducing the peak magnitude directly increases damping.

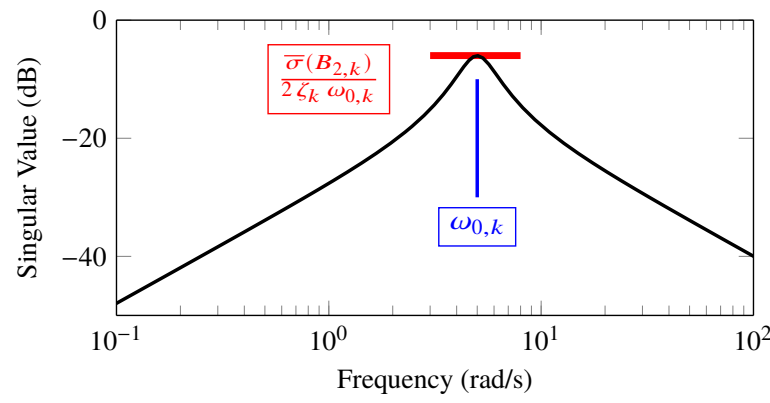

Fig. 10 Singular value of modal velocity $\hat{M}_{k}(s)$.

The transfer function (9) is an ideal description of damping requirements in the framework of $\mathcal{H}_{\infty}$-norm optimization: Increasing damping simply translates to flattening the peak and other specifications remain largely unaffected due to the narrow frequency band. Adding the modal velocity $\dot{\xi}_{k}$ as a performance output $p$ in Eq. (2) hence emphasizes, or "exposes", the specific dynamic mode within the minimization problem (4] [28, 29]. In case $B_{1, k} \neq[0 \cdots 0]$, the transfer function from $v$ to $\dot{\xi}_{k}$ retains very similar properties, but has a non-vanishing steady-state gain.

It remains to briefly discuss how the matrix $C_{p}$ in Eq. (2) is selected to achieve $p=\dot{\xi}_{k}$. Let $\varphi_{k} A=\lambda_{k} \varphi_{k}$. That is, $\varphi_{k} \in \mathbb{C}^{1 \times n_{x}}$ is a normalized left eigenvector and $\lambda_{k} \in \mathbb{C}$ is the corresponding eigenvalue for the $k^{\text {th }}$ complex mode. The generalized displacement $\xi$ and the generalized velocity $\dot{\xi}$ of the $k^{\text {th }}$ mode can be calculated as

$$
\begin{aligned}
& \xi_{k}=\operatorname{Im}\left(\varphi_{k}\right) x \\
& \dot{\xi}_{k}=\left(\operatorname{Re}\left(\lambda_{k}\right) \operatorname{Im}\left(\varphi_{k}\right)-\operatorname{Im}\left(\lambda_{k}\right) \operatorname{Re}\left(\varphi_{k}\right)\right) x .
\end{aligned}
$$

This fact is readily verified by using Eq. (11) as a state transformation and comparing the resulting state space realization with Eq. (8). To add $\dot{\xi}_{k}$ as a performance output $p$, the matrix $C_{p}$ in Eq. (2) is simply selected in accordance with Eq. (11b) and no state transformation is performed.

\section{Test Aircraft}

The aircraft under consideration in this paper is the University of Minnesota's mini MUTT, specifically the third mini MUTT built named "Geri". It is a remote-piloted flying wing aircraft with a wing span of $3 \mathrm{~m}$ and an approximate total mass of about $m=6.7 \mathrm{~kg}$. The mini MUTT is a laser-scanned replica of Lockheed Martin's Body Freedom Flutter 
vehicle [45] and resembles NASA's X56 MUTT aircraft [18, 46]. A schematic of the aircraft with sensor and flap positions is shown in Fig. 11. It is a low-speed swept-back flying wing with winglets on the wing tips for directional stability. An electric motor drives a pusher propeller mounted at the top rear of the rigid center body. The mini MUTT is specifically designed to exhibit strong coupling of rigid body and structural dynamics at low airspeed. Its wing structure consists of a solid spar and foam covered by a composite skin. The center body also has a composite structure. The entire trailing edge of the wing consists of eight control surfaces, two on the center body $\left(L_{1}, R_{1}\right)$ and six on the wings $\left(L_{2}, \ldots, L_{4}, R_{2}, \ldots, R_{4}\right)$. The aircraft is equipped with a rate gyro in the center body that measures the rotational rates $\omega$. Six accelerometers measure vertical accelerations $a$ in the center body (subscript C) and on both wing tips (subscripts L,R). These accelerometers are mounted in pairs, one fore (subscript $\mathrm{f}$ ) and one aft (subscript a).

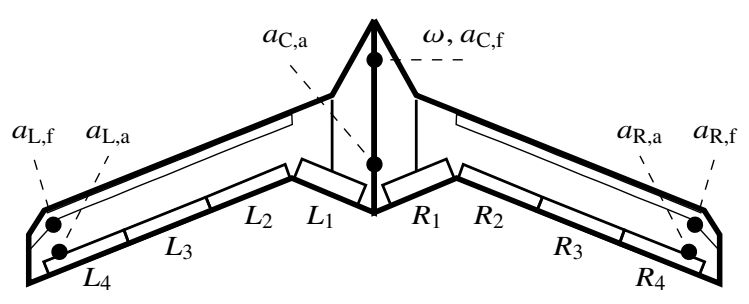

Fig. 11 Schematic of the mini MUTT aircraft.

\section{A. Aeroelastic Model}

A mathematical model of the aircraft was developed based on a mean axis approach as described in [47]. Mean axes are an attractive and widely used approach to model aeroelastic systems, specifically for control design. They offer the following advantages: (1) It is easy to incorporate nonlinear rigid-body dynamics, (2) the aerodynamics can be readily written in terms of dimensional aerodynamic derivatives, and (3) the format of the model is similar to a classical rigid-body flight dynamics model. A detailed description of mean axis models can be found in [47] based on a Lagrangian derivation. More recently, the mean axis formulation was also developed using a Newtonian approach in [48].

Mean axes form a body-referenced frame that is defined in the following way: The mean axis frame has no internal translational and angular momentum. Moreover, the origin of the frame is located at the instantaneous center of mass. Effectively, the mean axis are equivalent to the body frame for rigid bodies. However, it is important to emphasize that for a flexible body, the mean axis are a body-referenced frame but not a body-fixed frame. The following assumptions, commonly satisfied for aircraft [49], are further used to simplify the model: (1) The moments of inertia of the elastic body equal the moments of inertia of the undeformed body and are constant. (2) All elastic displacements occur primarlily in the same direction within the body frame, i.e., the cross product of displacements and displacement rates is almost zero. In [50], this is refered to as the practical mean axis constraint. (3) All deformations are small. 
Using these assumptions, the equations of motion of the flexible UAV can be written as

$$
\begin{aligned}
& m \ddot{r}_{\mathrm{r}}+\dot{r}_{\mathrm{r}} \times \omega_{\mathrm{r}}=F_{\mathrm{ext}} \\
& J_{\mathrm{r}} \dot{\omega}_{\mathrm{r}}+\omega_{\mathrm{r}} \times\left(J_{\mathrm{r}} \omega_{\mathrm{r}}\right)=M_{\mathrm{ext}} \\
& \mathcal{M}_{\mathrm{e}} \ddot{\eta}_{\mathrm{e}}+\mathcal{D}_{\mathrm{e}} \dot{\eta}_{\mathrm{e}}+\mathcal{K}_{\mathrm{e}} \eta_{\mathrm{e}}=\mathcal{F}_{\mathrm{e}} .
\end{aligned}
$$

Equation 12a represents the rigid-body translational dynamics with $\dot{r}_{\mathrm{r}}$ denoting the rigid-body velocities and $F_{\text {ext }}$ the external forces. Equation (12b) describes the rotational rigid-body dynamics where $\omega_{\mathrm{r}}$ are the rigid-body angular rates, $M_{\text {ext }}$ the external moments. The inertia tensor is assumed to be identical to the inertia $J_{\mathrm{r}}$ of the undeformed rigid body. Equation $12 \mathrm{c}$ gives the linear equations of motion for the elastic structural deformation in modal coordinates.

The elastic modal displacements are denoted $\eta_{\mathrm{e}}$ and the elastic modal forces $\mathcal{F}_{\mathrm{e}}$. The structural modal mass matrix, damping matrix, and stiffness matrix are denoted $\mathcal{M}_{\mathrm{e}}, \mathcal{D}_{\mathrm{e}}$ and $\mathcal{K}_{\mathrm{e}}$, respectively. These matrices and the corresponding geometric mode shapes for the six lowest-frequency elastic free-vibration modes are obtained from a finite element model and ground vibration tests [51]. The considered aircraft has an asymmetric wing stiffness due to manufacturing deficits. This fact is clearly seen in the mode shapes which are neither purely symmetric nor antisymmetric. The mode shapes of the first two elastic free-vibration modes are depicted in Figs. 12 and 13 . The first mode is dominantly symmetric and results mostly in bending deformation, although there is also notable twist deformation. The second mode is dominantly antisymmetric and causes mostly twist deformation with only the right wing bending. The asymmetric wing stiffness and the resulting asymmetric deformations lead to a more challenging control problem, as a combination of wing bending, wing twist, roll, and pitch dynamics is involved in the flutter phenomena.

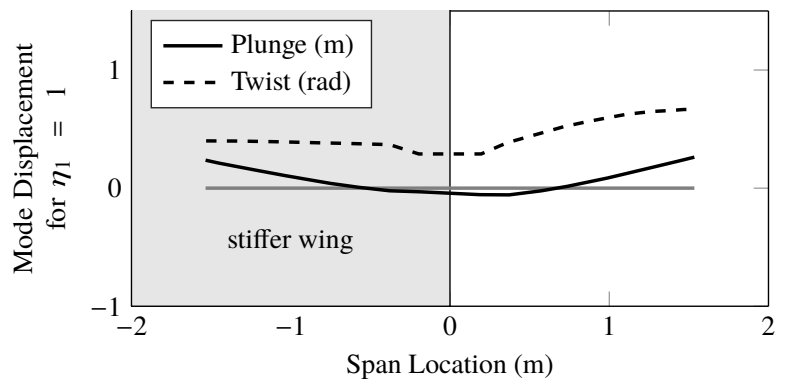

Fig. 12 Mode shape geometry of the first structural mode [52].

External forces are due to propulsion, gravity and aerodynamics, i. e., $F_{\text {ext }}=F_{\text {prop }}+F_{\text {grav }}+F_{\text {aero }}$. The only external moments considered in the model are of aerodynamic nature such that $M_{\mathrm{ext}}=M_{\text {aero }}$. The elastic modal forces $\mathcal{F}_{\mathrm{e}}$ are also due to aerodynamic effects. Since the aircraft only operates at small angles of attack $\left(\leq 10^{\circ}\right)$ at low airspeeds (i.e., in the incompressible flow regime), a simple linear aerodynamic model based on dimensional aerodynamic derivatives 


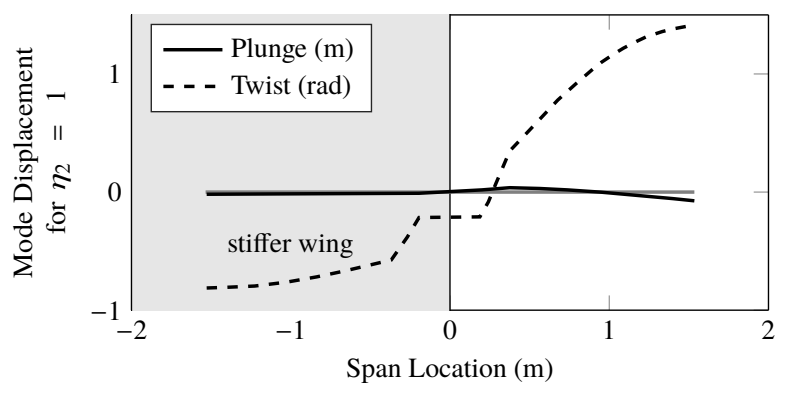

Fig. 13 Mode shape geometry of the second structural mode [52].

is used. The aerodynamic forces and moments can be written as

$$
\begin{aligned}
{\left[\begin{array}{c}
F_{\text {aero }} \\
M_{\text {aero }}
\end{array}\right]=A_{\mathrm{rr}}\left[\begin{array}{c}
\dot{r}_{\mathrm{r}} \\
\omega_{\mathrm{r}}
\end{array}\right]+A_{\mathrm{re}}\left[\begin{array}{c}
\dot{\eta}_{\mathrm{e}} \\
\eta_{\mathrm{e}}
\end{array}\right]+A_{\mathrm{rc}} \delta, } \\
\mathcal{F}_{\mathrm{e}}=A_{\mathrm{er}}\left[\begin{array}{c}
\dot{r}_{\mathrm{r}} \\
\omega_{\mathrm{r}}
\end{array}\right]+A_{\mathrm{ee}}\left[\begin{array}{c}
\dot{\eta}_{\mathrm{e}} \\
\eta_{\mathrm{e}}
\end{array}\right]+A_{\mathrm{ec}} \delta,
\end{aligned}
$$

where the matrices $A_{\mathrm{rr}}, A_{\mathrm{re}}, A_{\mathrm{er}}, A_{\mathrm{ee}}, A_{\mathrm{rc}}$ and $A_{\mathrm{ec}}$ contain dimensional aerodynamic coefficients and $\delta$ represents the deflection angles of the eight flaps. Initial aerodynamic coefficients were obtained using strip theory [53]. These values were then refined in a system identification campaign [52, 54]. Note that the dimensional aerodynamic coefficients are functions of the airspeed.

\section{B. Linearized Model}

For the following control design and linear analysis, the aircraft model is trimmed for steady-level flight at airspeeds ranging from $25 \mathrm{~m} / \mathrm{s}$ to $45 \mathrm{~m} / \mathrm{s}$ and an analytical linearization is performed. The result is a parametric linear system representation in dependence on the true airspeed $V_{\infty}$ which can be written as

$$
\begin{aligned}
& \dot{x}=A\left(V_{\infty}\right) x+B\left(V_{\infty}\right) \delta \\
& y=C\left(V_{\infty}\right) x+D\left(V_{\infty}\right) \delta .
\end{aligned}
$$

The state vector $x(t) \in \mathbb{R}^{18}$ of the parametric model contains the six rigid-body states (three translational and three rotational) as well as six generalized displacements and six generalized velocities for the structural modes. The output $y(t) \in \mathbb{R}^{9}$ represents the available sensor signals, i. e., the six vertical acceleration measurements $a$ and the three angular rates $\omega$ at the center of gravity. The input $\delta(t) \in \mathbb{R}^{8}$ represents the eight control signals for deflecting the flaps. All inputs and outputs are shown in Fig. 11 
The dynamics of the airframe are depicted in Fig. 14 in the form of a root locus with respect to airspeed. Note that the poles correspond to dynamic modes of the aircraft, i. e., they represent dynamic behavior that involves both rigid-body and elastic state variables. The corresponding dynamic mode shapes are shown in Fig. 15 in the form of phasor diagrams of the respective eigenvectors, normalized by the largest component. These diagrams show the phase and magnitude relations of the dominant state variables coupled in a dynamic mode, see e. g. [49]. Note that these relations are shown for $33 \mathrm{~m} / \mathrm{s}$, but also change with airspeed. The pole map shows several conventional modes such as the phugoid, dutch roll and roll subsidence mode. For example, the phasor diagram for the roll subsidence mode is dominated by the roll-rate state, see the upper left subplot of Fig. 15. The pole map also shows the short period mode. For conventional rigid aircraft, the short period dynamics only involve the vertical velocity $w$ and the pitch rate $q$, usually with $q$ leading $w$ by about $90^{\circ}$. The short period mode of the mini MUTT, however, involves also the structural deformation velocity $\dot{\eta}_{1}$, see the upper right subplot of Fig. 15 There are further six aeroelastic modes, four of which are outside of the depicted frequency range in Fig. 14 These modes can be traced back to originate in the six structural modes; their "genesis modes" for zero airspeed [15]. The two aeroelastic modes shown in Fig. 14, labeled $1^{\text {st }}$ AE and $2^{\text {nd }} \mathrm{AE}$, are critical for the flutter suppression control design. Their frequency and damping variation with airspeed is shown in Fig. 16 The first instability occurs at $33.3 \mathrm{~m} / \mathrm{s}$ with a frequency of $35.6 \mathrm{rad} / \mathrm{s}$. The second aeroelastic mode becomes unstable at $43.6 \mathrm{~m} / \mathrm{s}$ with a frequency of $20 \mathrm{rad} / \mathrm{s}$. The phasor diagrams in Fig. 15 show that the aeroelastic modes are dominated by the structural modes. Recalling the mode shape geometries shown in Figs. 12 and 13 , the first aeroelastic mode is primarily driven by wing bending while the second aeroelastic mode is mostly a torsion mode, but also involves bending. Consequently, instability of the first aeroelastic mode manifests itself as body-freedom-flutter with mostly symmetric wing bending and pitch motion, while instability of the second aeroelastic mode occurs as torsion flutter and roll motion.

\section{Phase Loss Modeling}

An important aspect of control-oriented modeling is an accurate description of the time delays and phase loss in the system. While also important for classical rigid-body flight control design, this aspect is absolutely essential for flutter suppression control design. Active flutter suppression operates at much higher frequencies than classical flight control systems and hence larger phase loss due to time delay and parasitic actuator and sensor dynamics occurs. For the aircraft under consideration, the two critical modes reside in a frequency range of $15-40 \mathrm{rad} / \mathrm{s}$. The rate measurements on the mini MUTT aircraft are obtained by an inertial measurement unit (IMU) that includes a $50 \mathrm{~Hz}$ low-pass filter. The accelerometer signals are filtered by an analog first-order low-pass filter with a bandwidth of $35 \mathrm{~Hz}$. These components are modeled by two transfer functions

$$
G_{\mathrm{acc}}(s)=\frac{2 \pi 35}{s+2 \pi 35} \quad \text { and } \quad G_{\mathrm{IMU}}(s)=\frac{2 \pi 50}{s+2 \pi 50} .
$$




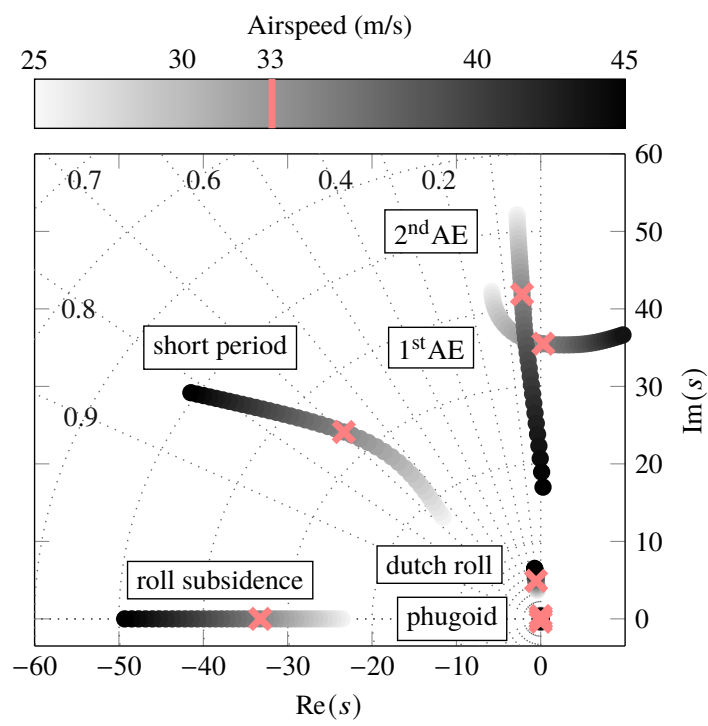

Fig. 14 Pole migration of the model with flutter occurring at $33.3 \mathrm{~m} / \mathrm{s}$ airspeed.

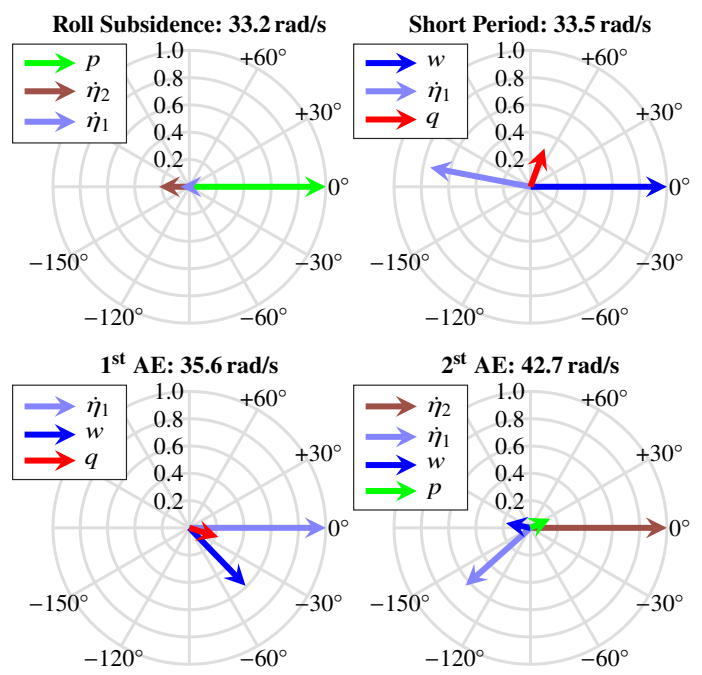

Fig. 15 Phasor diagrams of dynamic modes at $33.3 \mathrm{~m} / \mathrm{s}$ airspeed.

The signals provided by the sensors are processed by the mini MUTT's flight computer that executes the control algorithm within a $6.6 \mathrm{~ms}$ frame $(\approx 151 \mathrm{~Hz})$. The controller output is passed on to a microcontroller that runs asynchronously with a $3.3 \mathrm{~ms}$ frame rate $(\approx 302 \mathrm{~Hz})$ to generate a pulse width modulation (PWM) signal. This PWM signal is the input to a servo controller that runs, also asynchronously, with a $3.3 \mathrm{~ms}$ frame rate. The actuator used on the mini MUTT is a Futaba S9254 servo whose dynamics were identified using a chirp input signal and frequency-domain system identification. Its bandwidth (as defined by $45^{\circ}$ phase loss) is $100 \mathrm{rad} / \mathrm{s}$. The actuator dynamics and a delay of $25 \mathrm{~ms}$ (13.2 ms computation time, $6.6 \mathrm{~ms}$ zero-order hold delay, $5 \mathrm{~ms}$ actuator delay) are modeled jointly as a second-order 

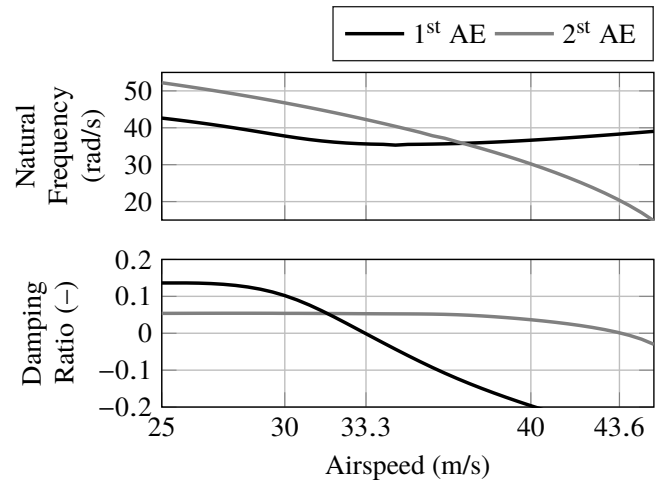

Fig. 16 Modal frequency and damping over airspeed.

transfer function

$$
G_{\text {act }}(s)=\frac{0.5583 s^{2}-107 s+7632}{s^{2}+139.9 s+7632}
$$

that accurately captures the behavior up to about $200 \mathrm{rad} / \mathrm{s}$. The phase loss introduced by $G_{\text {act }}(s)$ is already $60^{\circ}$ at a frequency of $30 \mathrm{rad} / \mathrm{s}$, which clearly shows the importance of including it in the design model.

\section{Control Design and Evaluation}

The following flutter suppression control design aims at maximizing the closed-loop flutter speed. This objective must be achieved while remaining within actuator limits. Given the catastrophic consequences of flutter, the controller must achieve sufficient robustness margins across all airspeeds. Finally, minimal interference with the autopilot is desired.

The autopilot uses the midboard flaps $\left(L_{3}, R_{3}\right)$ like a classical elevator for pitch attitude control and the inboard flaps $\left(L_{2}, R_{2}\right)$ like classical ailerons for roll attitude control and is similar to the one described in [55]. Consequently, the flutter suppression controller is assigned full authority over the body flaps and outboard flaps, i. e. $L_{1}, R_{1}, L_{4}$, and $R_{4}$ (in rad). Keeping the flutter suppression control loop completely separate from the autopilot loops reduces the risk of saturating the control surfaces and facilitates a simple control design. The body flaps have almost no effect on pitch, but produce sufficient lift to affect bending and twist of the structure. The outboard flaps have a strong effect on wing bending and twist as well as on pitch and roll. This choice of control surfaces leads to high functional controllability with effectors that can be combined in ways that isolate the two modes. The measured center-body pitch rate and roll rate (both in $\mathrm{rad} / \mathrm{s}$ ), and the vertical accelerations (in $\mathrm{ft} / \mathrm{s}^{2}$ ) as measured by the six accelerometers are used as feedback signals for the flutter suppression controller.

In order to maximize the flutter envelope expansion, several design airspeeds were considered. It was observed that the controllers designed at different airspeeds tend to stabilize the system up to their respective design airspeed, but not far beyond. This observation is consistent with previous research which showed that the design speed is an important 
design parameter [56]. Thus, a design airspeed as high as possible should be used. The frequency of the unstable first aeroelastic mode increases with higher airspeed. The maximum airspeed is hence limited by the available actuator bandwidth, since the bandwidth must be sufficiently higher than the frequency of the unstable mode [32, 57]. Keeping model accuracy and actuator limitations in mind, it is decided to restrict control authority to a maximum frequency of about $70 \mathrm{rad} / \mathrm{s}$. Consequently, an airspeed $V_{\infty}=41 \mathrm{~m} / \mathrm{s}$ is selected as the design condition. At this airspeed, the frequency of the unstable first aeroelastic mode ( $1^{\text {st }} \mathrm{AE}$ in Fig. 14 is $37 \mathrm{rad} / \mathrm{s}$, a factor two below the dedicated control bandwidth. The second aeroelastic mode ( $2^{\text {nd }}$ AE in Fig. 14 is stable, but lightly damped at a frequency of $28 \mathrm{rad} / \mathrm{s}$.

\section{A. Weight Design and Controller Tuning}

An airframe model with reduced state vector is used to form the plant model $P_{y}(s)$. Only the pitch and roll dynamics (represented by vertical velocity, pitch rate, and roll rate) as well as the first four structural modes are included. The $5^{\text {th }}$ and $6^{\text {th }}$ structural modes (at 180 and $200 \mathrm{rad} / \mathrm{s}$ ) have very little influence on the frequency range of interest and are ignored. The airframe model is combined with the models for sensor dynamics, actuator dynamics, and time delay as described in Sec. III.C. Finally, the generalized velocities $\dot{\xi}_{1}$ and $\dot{\xi}_{2}$ of the first two aeroelastic modes are added as performance outputs $p$ using Eq. (11b) as $C_{p}$ in Eq. (2).

The weights for the generalized plant (4) are selected as follows. Tracking is not an objective for the controller, so no frequency-dependent weight $W_{e}(s)$ is required and $W_{e}=0.5 I$ is selected. Similarly, $W_{p}=I$ is used, as the definition of $p$ as modal velocities already targets the specific narrow frequency range of the aeroelastic modes. Next, $W_{u}(s)=\frac{s+10}{s+0.1} \frac{s+70}{0.01 s+70} I$ is selected such that all control surfaces are assigned the frequency range 10-70 rad/s for activity. The wash-out minimizes the interaction with low-frequency dynamics and the autopilot. The roll-off provides robustness against inevitable model uncertainty at high frequencies.

Once these specifications are fixed, the static weights are used for controller tuning. The matrix $V_{e}$ is used to normalize the outputs such that an error of $1 \mathrm{rad} / \mathrm{s}$ pitch and roll rate is equally important as an acceleration error of $32 \mathrm{ft} / \mathrm{s}^{2} \approx 1 g$. Thus, $V_{e}=\operatorname{diag}(1,1,32,32,32,32,32,32)$ is selected. The control effort weight $V_{u}$ is initially selected to specify a maximum deflection of $3^{\circ}$ for all control surfaces in response to these errors, i. e., $V_{u}=\operatorname{diag}\left(\frac{3 \pi}{180}, \frac{3 \pi}{180}, \frac{3 \pi}{180}, \frac{3 \pi}{180}\right)$. To emphasize that robustness is a key concern, $V_{d}=V_{u}$ is selected, representing maximum input uncertainty at all inputs. The desired increase in modal damping is set by the weight $V_{p}$. Smaller values result in larger damping augmentation, since the weight encourages the controller to reduce the sharp peak in the transfer function from $d$ to $p$ more. Consequently, damping is increased in accordance with Eq. 10]. A weight $V_{p}=\operatorname{diag}(0.15,0.20)$ is selected such that the peaks of the open-loop frequency responses from $w_{2}$ to $z_{3}$ extend above the $0 \mathrm{~dB}$ line and hence contribute to the optimization objective, see Sec. IV.C. In summary, the total number of tuning parameters for the design is 19. Besides the two parameters that determine the frequency range of control activity and the single parameter to weight input disturbances, there is one tuning parameter for each of the eight measurement signals, four control effectors, and 
two modal velocity outputs. The number can be reduced to 8, when the tuning parameters $V_{e}$ and $V_{u}$ are not specified for each sensor and control effector individually, but instead for a group (one for all accelerometers, one for all rate sensors, one for all flaps).

Matlab's hinfsyn routine was used to synthesize a controller with these weights. A robustness analysis (see Sec. IV.B after the first controller synthesis showed unsatisfactory input margins at the right outboard flap $\left(R_{4}\right)$. This observation was attributed to the smaller stiffness of the right wing which effectively increases the control effectiveness on this side and hence the loop gain. To tackle the issue, the maximum deflection of $R_{4}$ in $V_{u}$ was iteratively adjusted to $V_{u}=\operatorname{diag}\left(\frac{3 \pi}{180}, \frac{3 \pi}{180}, \frac{3 \pi}{180}, \frac{1.6 \pi}{180}\right)$ in order to decrease the controller magnitude in the $R_{4}$ channel until satisfactory margins were obtained. Finally, the controller order was reduced from 35 to 25 . This optional step was performed to simplify implementation. The reduction was performed by transforming the controller to a modal form (7) and residualizing all dynamic modes with poles faster than $150 \mathrm{rad} / \mathrm{s}$. Doing so almost exactly preserved the frequency response of the controller up to about $100 \mathrm{rad} / \mathrm{s}$ and therefore did not cause any degradation in performance and robustness.

\section{B. Robustness Margins}

Every individual control loop, including autopilot loops for pitch and roll attitude hold, was verified to satisfy at least $45^{\circ}$ phase margin and $6 \mathrm{~dB}$ gain margin up to the design airspeed of $41 \mathrm{~m} / \mathrm{s}$. For this analysis, both the inputs (before the control surface actuators) and outputs (after the sensors) were considered as loop break points. Such an analysis is stricter in its requirements than standard procedures in aerospace such as MIL-SPEC9490 or SAE-AS94900 that consider loop break points at the inputs, only. The lowest margins are $6.8 \mathrm{~dB}$ gain margin at input $L_{4}$ and $50^{\circ}$ phase margin at input $R_{4}$. The lowest delay margins are $37 \mathrm{~ms}$ at input $R_{1}$ and $14 \mathrm{~ms}$ at output $a_{\mathrm{L}, \mathrm{a}}$. Note that these margins are on top of all known parasitic dynamics and delays. However, considering only classical margins and single loops does not acknowledge the complexity of the problem at hand. Destabilizing combinations of gain, phase, and directional changes that independently are considered safe can easily be overlooked. Therefore, symmetric disk margins [58] are used as an additional metric for robustness verification. They account for simultaneous perturbation of gain and phase and can also be calculated with respect to simultaneous perturbations at multiple inputs and outputs. Hence, they avoid the pitfalls of classical single-loop gain and phase margins.

The lowest single-loop disk margin at the design speed is $6.5 \mathrm{~dB} \mid 39^{\circ}$ and is attained at the input $L_{4}$. Thus, no critical combination of gain and phase variation exists in any of the feedback loops. It was further verified that all margins increase with lower airspeed. Further, multivariable symmetric disk margins are calculated to also consider directionality. These margins account for simultaneous perturbation at all outputs (multi-output disk margin), all inputs (multi-input disk margin), as well as at all inputs and outputs (multi-input-multi-output disk margin) [58]. Figure 17] shows these multivariable margins as a function of airspeed. As expected, their values are significantly lower than their single-loop counterparts. All margins decrease only slightly with increasing airspeed up until the design point $41 \mathrm{~m} / \mathrm{s}$. Therefore, no 
particular bottleneck can be identified. Beyond the design speed, the margins collapse and instability occurs at $42.5 \mathrm{~m} / \mathrm{s}$.

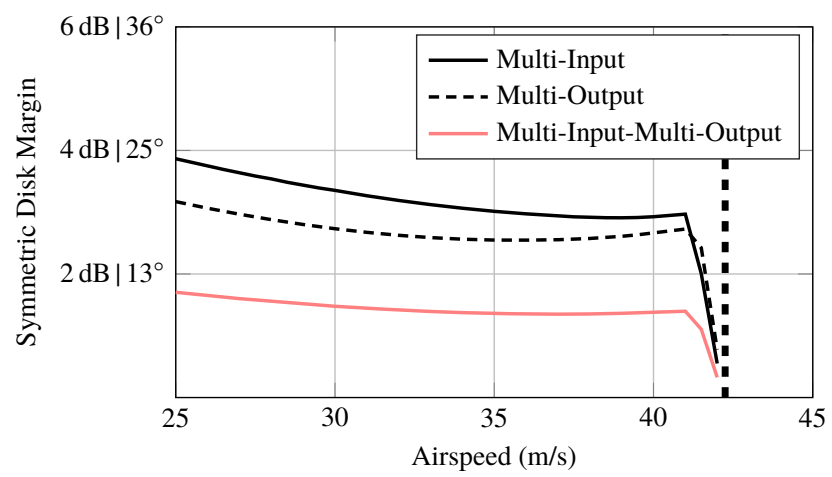

Fig. 17 Multi-loop disk margins.

\section{Results}

First, the effect of including the modal velocities as performance outputs is evaluated at the design point $V_{\infty}=41 \mathrm{~m} / \mathrm{s}$. The weighted load disturbance sensitivities of the first and second aeroelastic mode, i. e, the transfer functions from $w_{2}$ to $z_{3}$, are depicted in Figs. 18 and 19 . Stabilizing an unstable system inevitably leads to a net increase in sensitivity in accordance with Bode's sensitivity theorem [57]. Figure 18] shows this effect clearly for the first aeroelastic mode at the design airspeed. The magnitude of the frequency response of the stable closed-loop is increased compared to the unstable open-loop system. However, the increase is smallest at the mode's frequency such that the relative peak magnitude is actually decreased. Thus, a well-damped behavior of the closed loop can be expected. Figure 19 shows that the overall sensitivity degradation is much lower for the second aeroelastic mode. The peak magnitude is drastically decreased, both in absolute and relative terms. Thus, a large increase in damping compared to the open-loop system is achieved. The sensitivity degradation is well behaved and evenly spread out around the mode's frequency. In summary, the controller adds sufficient loop gain to stabilize the first mode without introducing a sensitivity peak and reduces the sensitivity peak in the second mode.

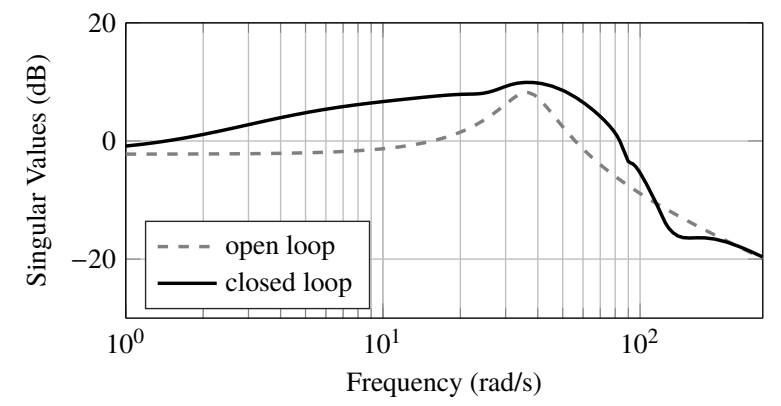

Fig. 18 Weighted load disturbance sensitivity ( $w_{2}$ to $z_{3}$ ) of modal velocity $\dot{\xi}_{1}$ at $41 \mathrm{~m} / \mathrm{s}$ airspeed. 


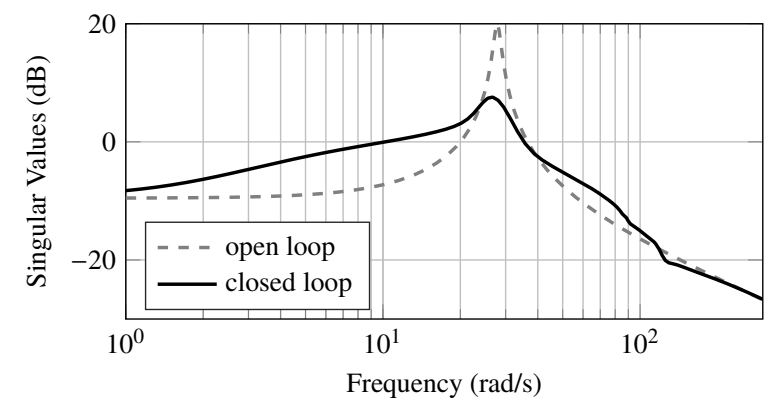

Fig. 19 Weighted load disturbance sensitivity $\left(w_{2}\right.$ to $\left.z_{3}\right)$ of modal velocity $\dot{\xi}_{2}$ at $41 \mathrm{~m} / \mathrm{s}$ airspeed.

Next, closed-loop pole migration with airspeed is evaluated. Recall from Fig. 14 that the open-loop model exhibits flutter at an airspeed above $33.3 \mathrm{~m} / \mathrm{s}$, indicated by the poles of the first aeroelastic mode crossing into the right half plane. Due to the presence of controller states $\left(x_{K}\right)$, the closed-loop system has more dynamic modes than the open-loop system. It is crucial to stress that these modes do not have a one-on-one correspondence with the dynamic modes of the open-loop system, even though part of the pole migration pattern shown in Fig. 20 resembles the one shown in Fig. 14 This becomes evident when the phasor diagrams of the modes are considered. Figure 21 shows the phasor diagrams of the four closed-loop modes labeled $M_{1}-M_{4}$ in Fig. 20 for $41 \mathrm{~m} / \mathrm{s}$ airspeed. Mode $M_{1}$ is dominated by the generalized velocity $\dot{\eta}_{2}$ of the second structural mode and the roll rate $p$. With torsion and roll being the main features, it is reminiscent of the second aeroelastic mode of the open-loop system. On the contrary, the mode $M_{2}$ is distinctly different from any open-loop mode. It consists of almost equal contributions from the generalized velocities $\dot{\eta}_{1}$ and $\dot{\eta}_{2}$ and further contains significant vertical velocity $(w)$ and pitch rate $(q)$ contributions. Both the modes $M_{3}$ and $M_{4}$ are largely dominated by the first structural mode, i. e., they represent mostly wing bending. Their phasor diagrams are almost identical, but neither resemble the short period, nor the first aeroelastic mode of the open-loop system. Focusing again on Fig. 20, it is interesting to observe that the damping of the closed-loop poles is near its maximum at the design speed. For lower airspeeds, the damping of $M_{2}$ decreases, while for larger airspeeds the damping of $M_{1}$ decreases. Instability occurs at $42.5 \mathrm{~m} / \mathrm{s}$, when the mode $M_{1}$ crosses into the right half plane. The phasor diagram for this airspeed qualitatively agrees with the one shown for $41 \mathrm{~m} / \mathrm{s}$ in Fig. 21. Hence, the instability is an asymmetric torsion roll flutter.

To gain additional insight, time-domain simulations are performed using a nonlinear model of the mini MUTT aircraft. The model contains all six elastic modes, first-order sensor dynamics and second-order actuator models that include deflection limits $\left(30^{\circ}\right)$, rate limits $\left(300^{\circ} / \mathrm{s}\right)$, and backlash $\left(1^{\circ}\right)$. The values for the actuator nonlinearities were measured and the $1^{\circ}$-backlash includes free-play of the linkage. Further, a $25 \mathrm{~ms}$ time-delay and the effects of a discrete controller implementation at a rate of $151 \mathrm{~Hz}$ are simulated. Figures 22 show data from a simulation with active flutter suppression. The scenario is a simultaneous pitch and roll attitude tracking task with references representative of a typical pilot input spectrum. The autopilot loops achieve satisfactory tracking performance throughout the simulation as shown in Fig. 22. They use less than $5^{\circ}$ inboard flap deflection $\left(L_{2}, R_{2}\right)$ and less than $2^{\circ}$ midboard flap deflection 


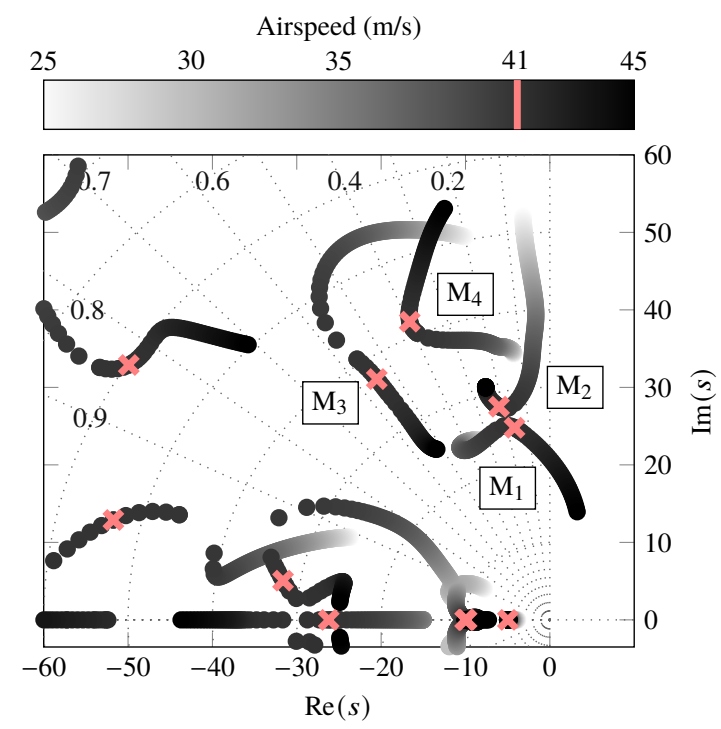

Fig. 20 Closed-loop pole migration with active flutter suppression control. The highlighted pole locations correspond to $41 \mathrm{~m} / \mathrm{s}$ airspeed.

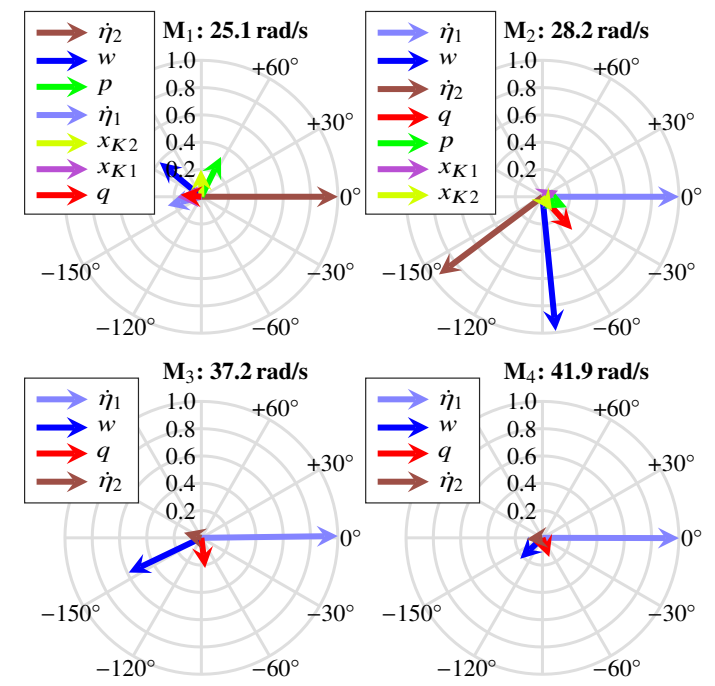

Fig. 21 Phasor diagram of closed-loop dynamic modes at $41 \mathrm{~m} / \mathrm{s}$ airspeed.

$\left(L_{3}, R_{3}\right)$ for this task. The resulting airspeed profile is depicted in Fig. 23 . The simulation starts slightly below the open-loop flutter speed of $33.3 \mathrm{~m} / \mathrm{s}$. During the tracking task, the airspeed exceeds this speed several times, up to $40 \mathrm{~m} / \mathrm{s}$. The time spend beyond the open-loop flutter speed is emphasized in Figs. 2324 through shaded areas.

Figure 24 shows that the control signals are so small that the backlash nonlinearity has a noticeable impact on the actual control surface deflections. In fact, the commanded deflections are only significant enough to overcome backlash when the aircraft is above its open-loop flutter speed. In the stable regime, the control signals are so small that they do not cause any change in deflection. It is interesting to note that the body flaps $L_{1}$ and $R_{1}$ are deflected purely symmetrically, while the outboard flaps $L_{4}$ and $R_{4}$ show some asymmetric behavior. Further, the right surface $R_{4}$ is 

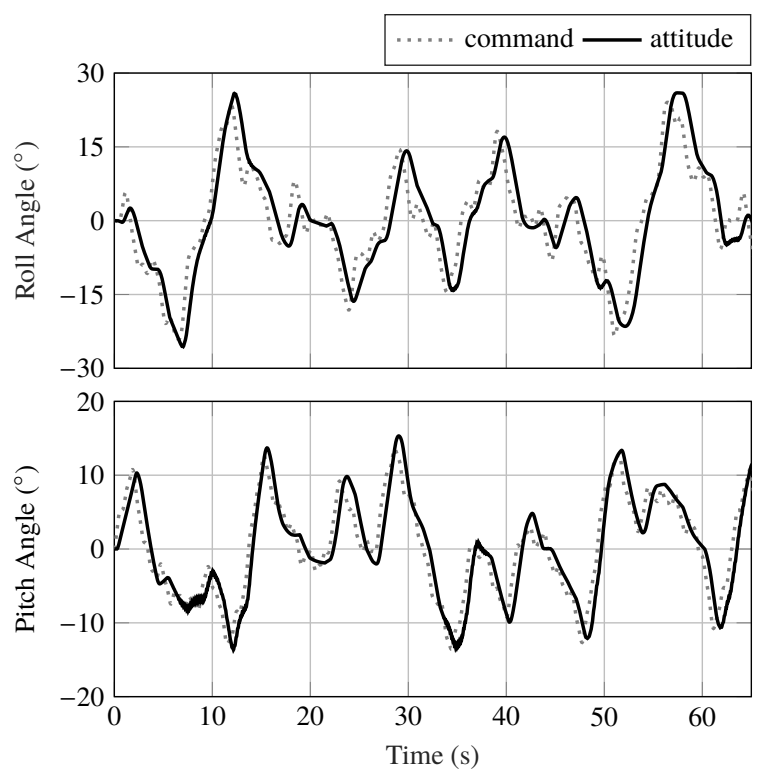

Fig. 22 Attitude tracking task.

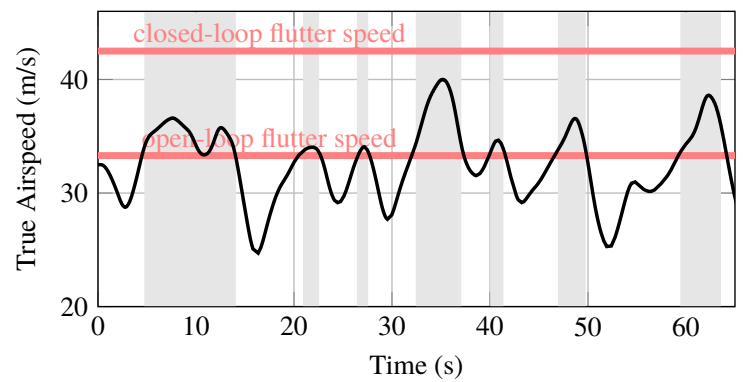

Fig. 23 True airspeed during attitude tracking task.

deflected significantly less than the left surface $L_{4}$ as intended by the choice of $V_{u}$ in the control design. Saturation is of no concern and the rate limit of the servo actuators was never violated during the simulation.

Finally, the aeroelastic response to a discrete gust as defined in FAA/EASA certification specification CS-25.341(a) is simulated. Figure 25 shows the displacement and twist of the right wing tip in response to a vertical gust. The gust has the form $\frac{5.66}{2}\left(1-\cos \left(\pi \frac{t-1}{0.31}\right)\right) \mathrm{m} / \mathrm{s}$ for $1 \leq t \leq 1.62$ and 0 for $t<0$ and $t>1.62$. The simulation is performed slightly below flutter speed to allow a comparison with and without active flutter suppression control. As is evident, the flutter suppression controller greatly reduces the structural oscillations.

\section{Conclusion}

This paper develops a systematic multivariable robust control design method that incorporates targeting of specific dynamic modes into the framework of closed-loop shaping. This is achieved by introducing additional performance outputs to the design model. These outputs represent generalized velocities in modal coordinates. Reducing the peaks in 

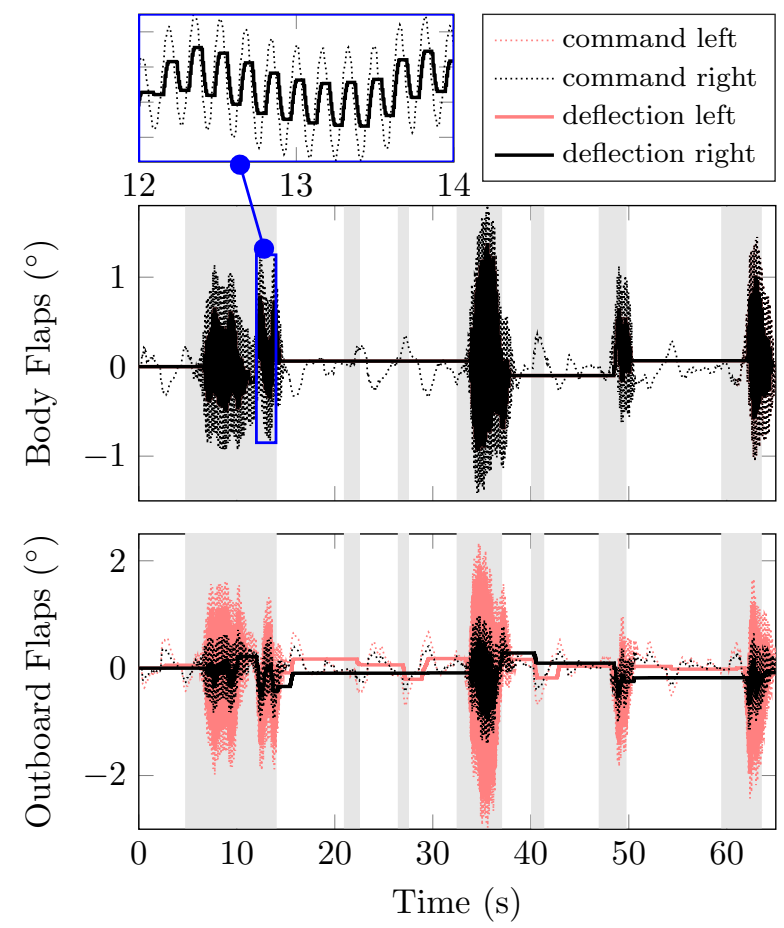

Fig. 24 Control surface deflections from flutter suppression controller during tracking task.
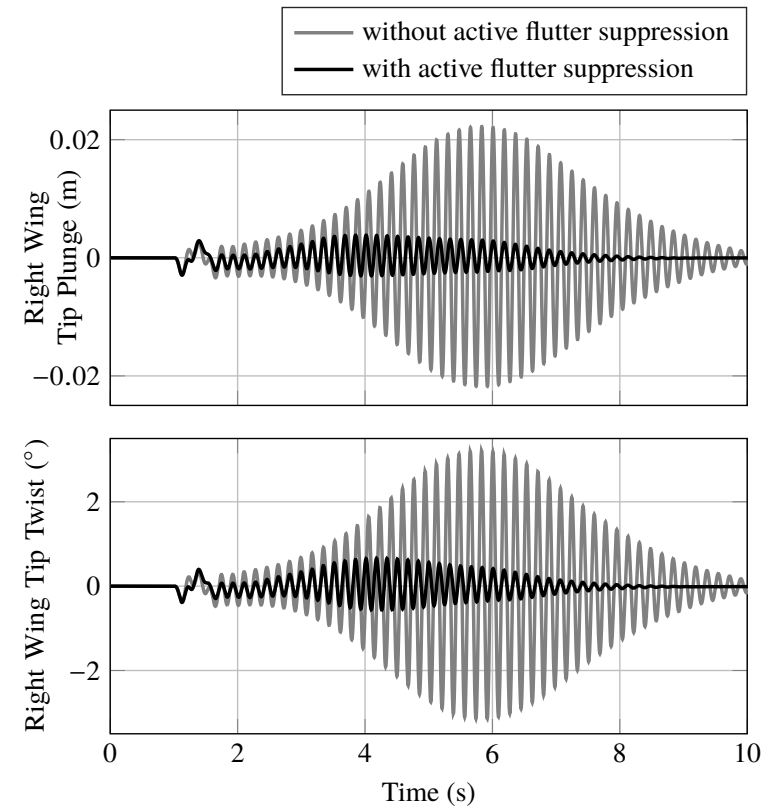

Fig. 25 Structural response to discrete vertical gust near the open-loop flutter speed $33.3 \mathrm{~m} / \mathrm{s}$.

their corresponding transfer functions is shown to directly relate to an increase in modal damping. The approach is used to increase the flutter speed of a small, unmanned flexible aircraft from $33.3 \mathrm{~m} / \mathrm{s}$ to $42.5 \mathrm{~m} / \mathrm{s}$. Asymmetry in the structural stiffness of the wings and large phase loss due to parasitic dynamics make this a particularly challenging 
control problem. The comprehensive design study demonstrates that the proposed method is ideally suited to design the flutter suppression controller in a way such that it targets the aeroelastic modes. The controller is tuned using a manageably low number of parameters that all have clear physical interpretations. It is shown that the design parameters indeed modify the controller in a transparent way, e. g., reduce the magnitude of a particular control input. Robustness analyses show that the controller achieves large classical gain and phase margins as well as reasonable multivariable disk margins across all airspeeds up to $41 \mathrm{~m} / \mathrm{s}$. That is, the feedback system is robust even close to the extended flutter speed. The controller is verified in simulation with actuator nonlinearities such as rate limits and backlash. Representative pilot inputs and discrete gusts are used as inputs. The control signals issued by the controller are only significant enough to exceed backlash above the open-loop flutter speed and overall remain small.

Future work could consider uncertain parameters in the model and generalize the method to incorporate uncertainty bounds at the design stage. Finally, the controller should be verified in flight tests. XXX

\section{Acknowledgement}

The authors gratefully acknowledge the contributions of our colleague and friend Gary Balas who initiated this work. The authors like to thank Brian Danowsky, Aditya Kotikalpudi, and Dave Schmidt for the prolonged collaboration on the topic and numerous helpful discussions. Thanks also goes to Lockheed Martin for donating the Body Freedom Flutter vehicle to the University of Minnesota. This vehicle was the starting point for the subsequent aircraft that were constructed and flight tested at Minnesota. Support for this work came from NASA NRA No. NNX14AL36A entitled Lightweight Adaptive Aeroelastic Wing for Enhanced Performance Across the Flight Envelope.

\section{References}

[1] Thompson, G. O., and Kass, G. J., “Active Flutter Suppression - An Emerging Technology,” Journal of Aircraft, Vol. 9, No. 3, 1972, pp. 230-235. URL https://doi.org/10.2514/3.58962

[2] Horikawa, H., and Dowell, E. H., “An Elementary Explanation of the Flutter Mechanism with Active Feedback Controls," Journal of Aircraft, Vol. 16, No. 4, 1979, pp. 225-232. URL https://doi.org/10.2514/3.58509

[3] Bendiksen, O. O., "Energy Approach to Flutter Suppression and Aeroelastic Control," Journal of Guidance, Control, and Dynamics, Vol. 24, No. 1, 2001, pp. 176-184. URL https://doi.org/10.2514/2.4699.

[4] Livne, E., "Aircraft Active Flutter Suppression: State of the Art and Technology Maturation Needs," Journal of Aircraft, Vol. 55, No. 1, 2018, pp. 410-452. URL https://doi.org/10.2514/1.c034442.

[5] Preumont, A., Vibration Control Of Active Structures, $2^{\text {nd }}$ ed., Kluwer Academic Publishers, New York, 2002.

[6] Wykes, J. H., "Structural Dynamic Stability Augmentation and Gust Alleviation of Flexible Aircraft," AIAA 5th Annual Meeting and Technical Display, 1968. URL https://doi.org/10.2514/6.1968-1067. 
[7] Wykes, J. H., Borland, C. J., Klepl, M. J., and MacMiller, C. J., "Design and Development of a Structural Mode Control System,” Contractor Report 143846, NASA, 1977.

[8] Wykes, J. H., Byar, T. R., MaeMiller, C. J., and Greek, D. C., "Analyses and Tests of the B-1 Aircraft Structural Mode Control System," Contractor Report 144887, NASA, 1980.

[9] Roger, K. L., Hodges, G. E., and Felt, L., “Active Flutter Suppression-A Flight Test Demonstration,” Journal of Aircraft, Vol. 12, No. 6, 1975, pp. 551-556. URL https://doi.org/10.2514/3.59833

[10] Schmidt, D. K., and Chen, T. K., "Frequency domain synthesis of a robust flutter suppression control law," Journal of Guidance, Control, and Dynamics, Vol. 9, No. 3, 1986, pp. 346-351. URL https://doi.org/10.2514/3.20112

[11] Luton, J. A., and Mook, D. T., "Numerical simulations of flutter and its suppression by active control," AIAA Journal, Vol. 31, No. 12, 1993, pp. 2312-2319. URL/https://doi.org/10.2514/3.11930.

[12] Waszak, M. R., and Srinathkumar, S., "Flutter Suppression for the Active Flexible Wing: A Classical Design," Journal of Aircraft, Vol. 32, No. 1, 1995, pp. 61-67. URL https://doi.org/10.2514/3.46684.

[13] Mukhopadhyay, V., "Flutter Suppression Control Law Design And Testing For The Active Flexible Wing," Journal of Aircraft, Vol. 32, No. 1, 1995, pp. 45-51. URL https://doi.org/10.2514/3.46682

[14] Mukhopadhyay, V., "Transonic Flutter Suppression Control Law Design and Wind-Tunnel Test Results,” Journal of Guidance, Control, and Dynamics, Vol. 23, No. 5, 2000, pp. 930-937. URL https://doi.org/10.2514/2.4635

[15] Schmidt, D. K., "Stability Augmentation And Active Flutter Suppression Of A Flexible Flying-Wing Drone," Journal of Guidance, Control, and Dynamics, Vol. 39, No. 3, 2016, pp. 409-422. URL https://doi.org/10.2514/1.G001484

[16] Newsom, J. R., "Control Law Synthesis for Active Flutter Suppression Using Optimal Control Theory,” Journal of Guidance and Control, Vol. 2, No. 5, 1979, pp. 388-394. URL https://doi.org/10.2514/3.55894

[17] Mahesh, J., Stone, C., Garrard, W., and Dunns, H., “Control Law Synthesis for Flutter Suppression Using Linear Quadratic Gaussian Theory," Journal of Guidance and Control, Vol. 4, No. 4, 1981, pp. 415-422. URL https://doi.org/10.2514/3. 56094

[18] Ryan, J. J., Bosworth, J. T., Burken, J. J., and Suh, P. M., "Current and Future Research in Active Control of Lightweight, Flexible Structures Using the X-56 Aircraft,” AIAA SciTech, 2014. URL https://doi .org/10.2514/6.2014-0597

[19] Barker, J. M., Balas, G. J., and Blue, P. A., “Gain-scheduled Linear Fractional Control for Active Flutter Suppression,” Journal of Guidance, Control, and Dynamics, Vol. 22, No. 4, 1999, pp. 507-512. URL https://doi.org/10.2514/2.4418

[20] Barker, J. M., and Balas, G. J., “Comparing Linear Parameter-Varying Gain-Scheduled Control Techniques For Active Flutter Suppression,” Journal of Guidance, Control, and Dynamics, Vol. 23, No. 5, 2000, pp. 948-955. URL https: //doi.org/10.2514/2.4637 
[21] Waszak, M. R., "Robust Multivariable Flutter Suppression for Benchmark Active Control Technology Wind-Tunnel Model," Journal of Guidance, Control, and Dynamics, Vol. 24, No. 1, 2001, pp. 147-153. URL https://doi .org/10.2514/2.4694

[22] Zhao, Y., "Flutter Suppression of a High Aspect-Ratio Wing with Multiple Control Surfaces," Journal of Sound and Vibration, Vol. 324, No. 3-5, 2009, pp. 490-513. URL https://doi.org/10.1016/j.jsv.2009.02.026

[23] Holm-Hansen, B., Atkinson, C., Benarek, J., Burnett, E., Nicolai, L., and Youssef, H., "Envelope Expansion of a Flexible Flying Wing by Active Flutter Suppression,” Proceedings of the Association for Unmanned Vehicle Systems International, 2010.

[24] Hjartarson, A., Seiler, P. J., and Balas, G. J., "LPV Aeroservoelastic Control using the LPVTools Toolbox," AIAA Atmospheric Flight Mechanics Conference, 2013. URL https://doi.org/10.2514/6.2013-4742

[25] Danowsky, B. P., Thompson, P. M., Lee, D., and Brenner, M., "Modal Isolation and Damping for Adaptive Aeroservoelastic Suppression," AIAA Atmospheric Flight Mechanics Conference, 2013. URL https://doi.org/10.2514/6.2013-4743

[26] Pusch, M., "Aeroelastic Mode Control using H2-optimal Blends for Inputs and Outputs," AIAA Guidance, Navigation, and Control Conference, 2018. URL https://doi.org/10.2514/6.2018-0618

[27] Pusch, M., Ossmann, D., and Luspay, T., "Structured Control Design for a Highly Flexible Flutter Demonstrator," Aerospace, Vol. 6, No. 3, 2019, p. 27. URL https://doi.org/10.3390/aerospace6030027

[28] Hanel, M., "Robust Integrated Flight and Aeroelastic Control System Design for a Large Transport Aircraft," Ph.D. thesis, University Stuttgart, Stuttgart, Germany, 2001.

[29] Theis, J., Pfifer, H., Balas, G., and Werner, H., "Integrated Flight Control Design for a Large Flexible Aircraft," American Control Conference, 2015, pp. 3830-3835. URL https://doi.org/10.1109/ACC.2015.7171927

[30] Theis, J., Pfifer, H., and Seiler, P., "Robust Control Design for Active Flutter Suppression,” AIAA Atmospheric Flight Mechanics Conference, 2016. URL https://doi.org/10.2514/6.2016-1751

[31] Zhou, K., Doyle, J. C., and Glover, K., Robust and Optimal Control, Prentice Hall, Upper Saddle River, NJ, 1995.

[32] Skogestad, S., and Postlethwaite, I., Multivariable Feedback Control, $2^{\text {nd }}$ ed., Prentice Hall, Upper Saddle River, NJ, 2005.

[33] Glover, K., and Doyle, J. C., "State-space Formulae for All Stabilizing Controllers that Satisfy an $\mathcal{H}_{\infty}$-norm Bound and Relations to Risk Sensitivity," Systems \& Control Letters, Vol. 11, No. 3, 1988, pp. 167-172. URL https://doi .org/10.1016/0167$6911(88) 90055-2$

[34] Doyle, J. C., Glover, K., Khargonekar, P. P., and Francis, B. A., "State-space Solutions to Standard $\mathcal{H}_{2}$ and $\mathcal{H}_{\infty}$ Control Problems," IEEE Transactions on Automatic Control, Vol. 34, No. 8, 1989, pp. 831-847. URL/https://doi.org/10.23919/ ACC. 1988.4789992

[35] Gahinet, P., and Apkarian, P., “A Linear Matrix Inequality Approach to $H_{\infty}$ Control," International Journal of Robust and Nonlinear Control, Vol. 4, No. 4, 1994, pp. 421-448. URL https://doi.org/10.1002/rnc.4590040403 
[36] Apkarian, P., and Noll, D., "Nonsmooth $H_{\infty}$ Synthesis," IEEE Transactions on Automatic Control, Vol. 51, No. 1, 2006, pp. 71-86. URL https://doi.org/10.1109/TAC.2005.860290

[37] Biannic, J., and Apkarian, P., "A new approach to fixed-order $H_{\infty}$ synthesis: Application to autoland design," AIAA Guidance, Navigation, and Control Conference, 2001.

[38] Falcoz, A., Pittet, C., Bennani, S., Guignard, A., Bayart, C., and Frapard, B., "Systematic Design Methods of Robust and Structured Controllers for Satellites," CEAS Space Journal, Vol. 7, No. 3, 2015, pp. 319-334. URLhttps://doi .org/10.1007/s12567015-0099-8

[39] Balas, G., Chiang, R., Packard, A., and Safonov, M., "Robust Control Toolbox R2019a,” Reference, The MathWorks, Inc., Natick, MA, 2019.

[40] Sefton, J., and Glover, K., "Pole/Zero Cancellations in the General $\mathcal{H}_{\infty}$ Problem with Reference to a Two Block Design," Systems \& Control Letters, Vol. 14, No. 4, 1990, pp. 295-306. URL https://doi .org/10.1016/0167-6911(90)90050-5

[41] Saupe, F., "Linear Parameter Varying Control Design for Industrial Manipulators," Ph.D. thesis, Hamburg University of Technology, Hamburg, Germany, 2013.

[42] Theis, J., "Robust and Linear Parameter-Varying Control of Aeroservoelastic Systems," Ph.D. thesis, Hamburg University of Technology, Hamburg, Germany, 2018. URL https://doi.org/10.15480/882.1635

[43] Bryson, A. E., and Ho, Y.-C., Applied Optimal Control, Hemisphere Pub. Corp., Washington, 1975.

[44] Müller, P. C., and Schiehlen, W. O., Linear Vibrations: A theoretical treatment of multi-degree-of-freedom vibrating systems, Springer, 1985.

[45] Beranek, J., Nicolai, L., Buonanno, M., Burnett, E., Atkinson, C., Holm-Hansen, B., and Flick, P., “Conceptual Design of a Multi-Utility Aeroelastic Demonstrator,” 13th AIAA/ISSMO Multidisciplinary Analysis Optimization Conference, $2010, \mathrm{pp}$. 2194-2208. URL https://doi.org/10.2514/6.2010-9350

[46] Burnett, E. L., Beranek, J. A., Holm-Hansen, B. T., Atkinson, C. J., and Flick, P. M., "Design and flight test of active flutter suppression on the X-56A multi-utility technology test-bed aircraft,” The Aeronautical Journal, Vol. 120, No. 1228, 2016, pp. 893-909. URL https://doi.org/10.1017/aer.2016.41.

[47] Milne, R. D., "Dynamics of the Deformable Aeroplane," Tech. Rep. 3345, Aeronautical Research Council Reports and Memoranda, 1964.

[48] Keyes, S. A., Seiler, P., and Schmidt, D. K., "Newtonian Development of the Mean-Axis Reference Frame for Flexible Aircraft," Journal of Aircraft, Vol. 56, No. 1, 2019, pp. 392-397. URL https://doi.org/10.2514/1.C035041

[49] Schmidt, D. K., Modern Flight Dynamics, McGraw-Hill, New York, 2012. 
[50] Waszak, M. R., and Schmidt, D. K., "Flight Dynamics of Aeroelastic Vehicles," Journal of Aircraft, Vol. 25, No. 6, 1988, pp. 563-571. URL https://doi.org/10.2514/3.45623.

[51] Gupta, A., Zhao, W., Regan, C., Seiler, P., and Kapania, R., "Identification of Symmetrical Structures with Fabrication and Damage Induced Asymmetry," International Conference on Experimental Vibration Analysis for Civil Engineering Structures, 2017,pp. 683-693. URL https://doi.org/10.1007/978-3-319-67443-8_60

[52] Danowsky, B. P., Schmidt, D. K., and Pfifer, H., "Control-Oriented System and Parameter Identification of a Small Flexible FlyingWing Aircraft," AIAA Atmospheric Flight Mechanics Conference, 2017. URL https://doi.org/10.2514/6.2017-1394

[53] Schmidt, D. K., Zhao, W., and Kapania, R. K., "Flight-Dynamics and Flutter Modeling and Analysis of a Flexible Flying-Wing Drone," AIAA Atmospheric Flight Mechanics Conference, 2016. URL https://doi .org/10.2514/6.2016-1748

[54] Pfifer, H., and Danowsky, B., "System Identification of a Small Flexible Aircraft," AIAA Atmospheric Flight Mechanics Conference, 2016. URL https://doi.org/10.2514/6.2016-1750

[55] Schmidt, D. K., Danowsky, B. P., Kotikalpudi, A., Seiler, P. J., and Kapania, R. K., "Flight Dynamics, Flutter Analysis, and Control of an MDAO Designed Flying Wing Research Drone,” AIAA Atmospheric Flight Mechanics Conference, 2019. URL https://doi.org/10.2514/6.2019-1816

[56] Kotikalpudi, A., Danowsky, B. P., Schmidt, D., Theis, J., and Seiler, P., "Flutter Suppression Control Design for a Small, Flexible Flying-Wing Aircraft," AIAA Aviation and Aeronautics Forum and Exposition, 2018. URL https://doi .org/10. $2514 / 6.2018-3426$

[57] Stein, G., "Respect the Unstable," IEEE Control Systems Magazine, 2003, pp. 12-25. URL https://doi.org/10.1109/ MCS.2003.1213600

[58] Blight, J. D., Lane Dailey, R., and Gangsaas, D., "Practical Control Law Design for Aircraft Using Multivariable Techniques," International Journal of Control, Vol. 59, No. 1, 1994, pp. 93-137. URLhttps://doi .org/10.1080/00207179408923071 